\title{
OCT Angiography (OCTA) in Retinal Diagnostics
}

\author{
Roland Rocholz, Federico Corvi, Julian Weichsel, \\ Stefan Schmidt, and Giovanni Staurenghi
}

\subsection{Introduction}

Optical coherence tomography angiography (OCTA) is a non-invasive imaging technique which can be used to provide three-dimensional visualization of perfused vasculature of the retina and choroid [1, 2]. In contrast to standard structural optical coherence tomography (OCT, see Chap. 5), OCTA analyzes not only the intensity of the reflected light but also the temporal changes of the OCT signal. Based on repeated OCT section images (B-Scans) from the same location of the retina, it is possible to separate the temporal signal changes caused by moving particles (such as erythrocytes flowing through vessels) from other sources of signal change (i.e. eye motion or noise in the OCT signal). Thereby image contrast between perfused vessels and static surrounding tissues can be created as illustrated in Fig. 6.1.

Using dense volume scans, it is possible to obtain OCTA images that are similar to fluorescence angiography images, which are the clinical

R. Rocholz $(\bowtie) \cdot J$. Weichsel $\cdot$ S. Schmidt Heidelberg Engineering $\mathrm{GmbH}$, Heidelberg, Germany

F. Corvi · G. Staurenghi

Eye Clinic, Department of Biomedical and Clinical Science "Luigi Sacco", Sacco Hospital, University of Milan, Milan, Italy gold standard. In contrast to fluorescence angiography, OCTA has the advantage of not requiring any dye injection. Moreover, while fluorescence angiography provides only two-dimensional images of the fundus, OCTA enables the visualization of structure and blood flow within the vitreous, the retina, and the choroid, separately (see Sects. 6.2.2 and 6.2.3). Using appropriately adjusted segmentation boundaries, it is also possible to examine the distinct capillary networks of the retina (with vessel diameters as small as approx. $8 \mu \mathrm{m}$ ) [3]. The definition of the separating boundaries has evolved since the introduction of OCTA in the clinical practice and is described in Sect. 6.2.4.

Various OCTA algorithms have been proposed and utilized in research and in clinical devices for OCTA image construction (see Sect. 6.2.1). Therefore OCTA images from different devices vary in appearance $[4,5]$, which may result in different clinical diagnostic interpretations. While each unique OCTA algorithm is subject to slightly different limitations that are attributed to its overall approach, there are certain confounding factors and/or limitations that impact all algorithms and are innate characteristics of this imaging modality [5]. These factors include, but are not limited to, reduced light penetration in deeper layers and image artifacts projected from more superficial layers to deeper ones. Artifacts can originate from image acquisition, eye motion, 

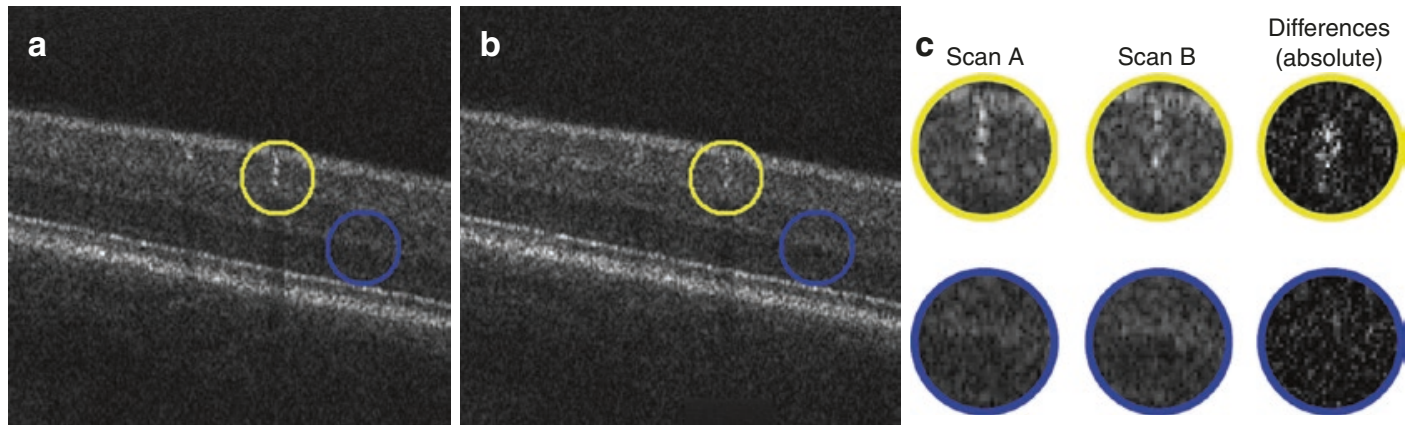

Fig. 6.1 Example of how the OCT signal intensity changes over time, after bulk motion correction. (a, b) Structural OCT images were acquired with a time difference of $8 \mathrm{~ms}$. The location of a larger blood vessel (yellow circle) and of static tissue (blue circle) is indicated in both

image processing, and display strategies [5]. Section 6.3 describes some of the major artifacts related to OCTA as well as state-of-the-art countermeasures.

Section 6.2 .5 briefly introduces OCTA metrics, which are intended for quantitative evaluation of OCTA data. Such numerical aggregates of the image data enable an objective analysis of disease progression and statistical conclusions in larger studies of diseases.

With fluorescence angiography, namely Fluorescein Angiography (FA) and Indocyanine Green Angiography (ICGA), dynamic phenomena such as dye leakage, pooling, and staining can additionally be observed. These phenomena cannot be observed with OCTA because no motion of blood cells is involved. While these phenomena are also used in clinical diagnosis [6], retinal pathology can also be obscured by leakage or hemorrhage. In contrast, OCTA can generate high contrast, well-defined images of the microvasculature below areas of leakage or hemorrhage [7]. Therefore, dye-based angiography and OCTA are giving complementary information. To illustrate the similarities and differences of OCTA with respect to the gold standard dye-based angiography, Sect. 6.4 provides side-by-side comparisons for clinical cases of diabetic retinopathy, retinal vein occlusion, macular telangiectasia, and age-related macular degeneration. images. (c) Upon magnification of these areas and calculation of the absolute differences, larger signal changes can be seen within the blood vessel compared to the static tissue

\subsection{Technical Foundation for Clinical OCTA Imaging}

OCT systems typically produce section images as shown in Fig. 6.1. Such images are commonly referred to as B-Scans. As can be seen in Fig. 6.1a, the B-Scans show a grainy pattern, also known as speckle pattern. These speckles are inherent to the interferometric OCT measurement. If two B-Scans are taken from the very same location of the retina (cf. Fig. 6.1a, b), the speckle pattern at locations of static tissue basically stays the same. In contrast, at locations of perfused blood vessels, the speckle pattern changes over time. The basic principle of OCTA is therefore to analyze the temporal variation of the OCT signal in order to derive an image of the perfused retinal vasculature.

To allow for the creation of images similar to fundus images from standard angiography, volume scans are performed. This means, that multiple adjacent B-Scans are acquired to cover extended regions of the retina. Eventually, these B-Scans are combined to form a three-dimensional sample of the retinal structure and blood flow.

\subsubsection{OCTA Signal Processing and Image Construction}

In OCTA imaging, several OCT B-Scans of the same retinal cross section are acquired repeatedly 
in short succession. Within this retinal cross section, at locations of static tissue, the microscopic configuration of illuminated scattering particles in the beam focus is well preserved over sequential acquisitions and consequently yields a consistent OCT signal over time. Contrarily, at locations where directed motion is present in the sample, like in retinal blood vessels, the scattering particles are continuously replaced by other particles in subsequent acquisitions. This continuous exchange of microscopic particles modulates the OCT signal and introduces an additional source of variability to the repeated measurements. Overall, the smaller the remaining portion of conserved scattering particles in the beam waist, the higher the variability in the OCT signal of sequential acquisitions. Maximum OCT signal variability is observed when the scattering particles are completely replaced by others in subsequent measurements. Clinical OCTA imaging on current commercially available OCT device hardware is typically operating in this regime, as the physiological blood flow speeds in most of the perfused retinal vasculature [8] significantly exceeds the velocity limit (i.e. typically only few $\mathrm{mm} / \mathrm{s}$ ) determined by the product of OCT beam waist diameter and scan repetition rate. Hence, it is unlikely to observe the same red blood cell configuration in two successive B-Scans. This, however, is a necessary requirement for deriving quantitative blood flow velocity measurements from the OCT signal variation in repeated scans (cf. Chap. 7). Accepting this current technical limitation of clinical OCT devices, OCTA algorithms for signal construction rather focus on reliably differentiating locations of significant blood flow from static tissue, instead of measuring blood flow velocity quantitatively. In this context, OCTA image construction is a quasibinary classification problem with the goal to optimally distinguish significant flow from static tissue at each location of the retina.

Different algorithmic strategies have been suggested in the past for optimally addressing this classification task. While some algorithms are using exclusively either amplitude or phase of the complex OCT signal, others are combining information from both. As the microscopic pattern of illuminated particles in the sample and its detailed configurational change according to motion is practically inaccessible to measurement, its influence on the resulting OCT signal is typically rather considered probabilistically as a stochastic contribution to the overall measurement. OCTA algorithms are thus quantifying the amount of variability in the random realizations of the measured OCT signal from repeated acquisitions in one or the other way. Practically, for instance the temporal correlation [3] or the overall variance [4] of the signal or other more involved statistical parameters [2] with an expected relation to the OCT signal variability are assessed in different approaches. These statistical parameters, potentially after additional post-processing and contrast enhancement, are subsequently taken as the resulting OCTA signal in arbitrary units. As an alternative to these statistical parameter based OCTA signal construction methods, underlying probabilistic statistical models for the random OCT signal from sample locations with and without directed flow can be derived from theory and experiment [9-11]. Based on these underlying models and the repeated OCT signal observations, a probability for being static (versus in flow) can be assigned to each measured location. This holds the advantage of yielding easily interpretable probability values and no further contrast enhancement of the resulting OCTA signal is needed.

\subsubsection{OCTA Data Visualization}

The acquisition of OCTA volume scans yields three-dimensional data of the retinal structure and blood flow. To visualize and analyze such rich datasets, different image representations are used, as illustrated in Fig. 6.2. To separately visualize the vascular networks (or plexuses) in the retina (cf. Sect. 6.2.4) and pathological alterations in normally avascular tissue, the review of OCTA data is typically based on so-called en face images that are generated from within slabs of the acquired volume. In this context, the term slab refers to a section of finite axial extent in the volumetric data delimited by an anterior and a posterior boundary surface. These slab boundaries are usually determined by layer 
Fig. 6.2 Visualization of OCT angiography data is mainly based on en face images and section images. (a) En face image of the within the superficial vascular plexus. In the background, an infrared cSLO fundus image is image of the corresponding OCTA data. (c1-c3): OCT/ OCTA fusion images of a section along the fast scanning axis (B-Scan direction, green). (c1) Section image shows structural OCT in the background and the OCTA data as yellow overlay. (c2) Same as (c1), but the structural OCT data is faded out. (c3) Same as (c1), but the OCTA data is faded out. (d) OCT/OCTA fusion image where section is along the slow scanning axis (orthogonal to B-Scan direction, blue) structural OCT data shown. (b) En face
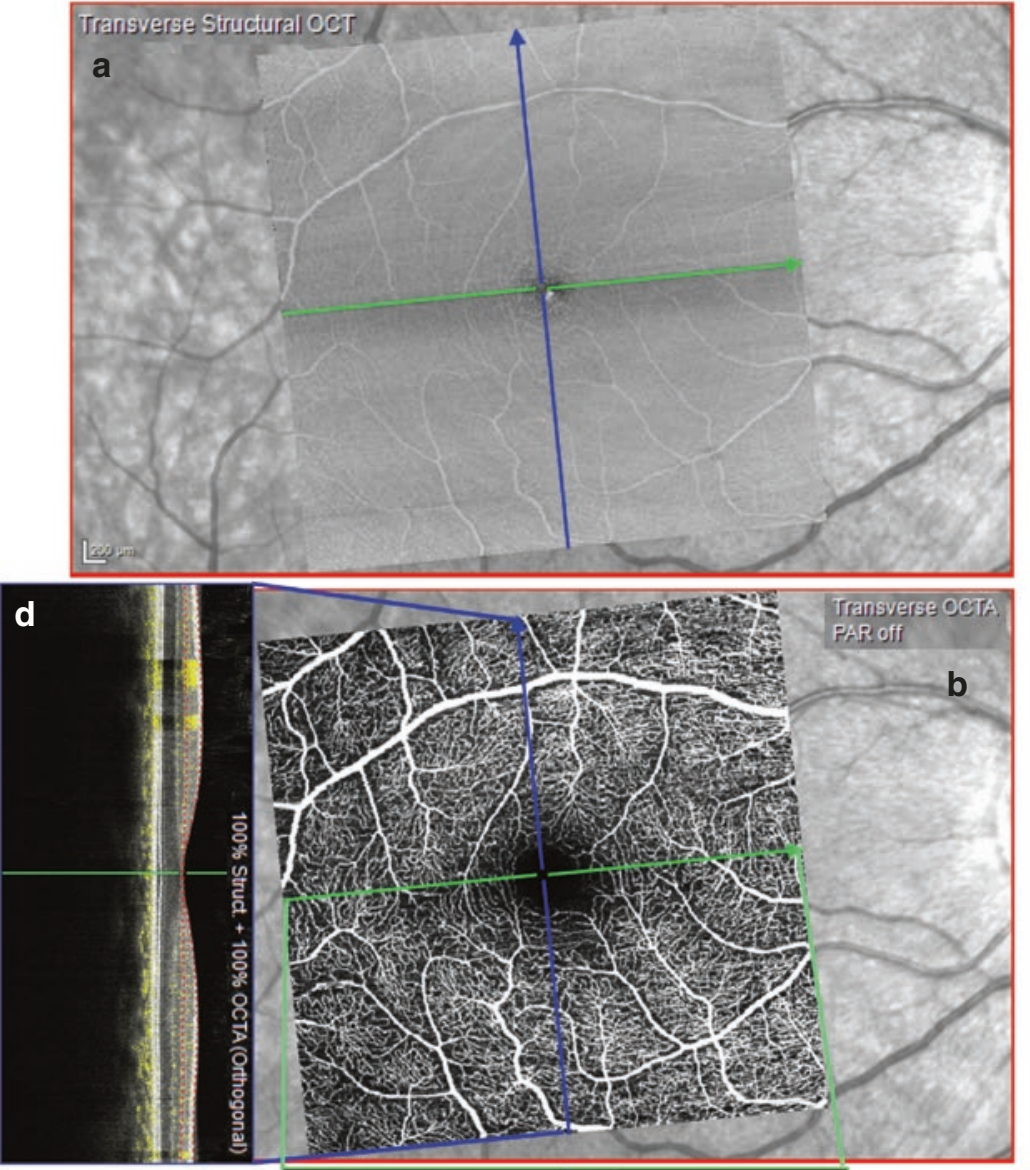

\section{d}

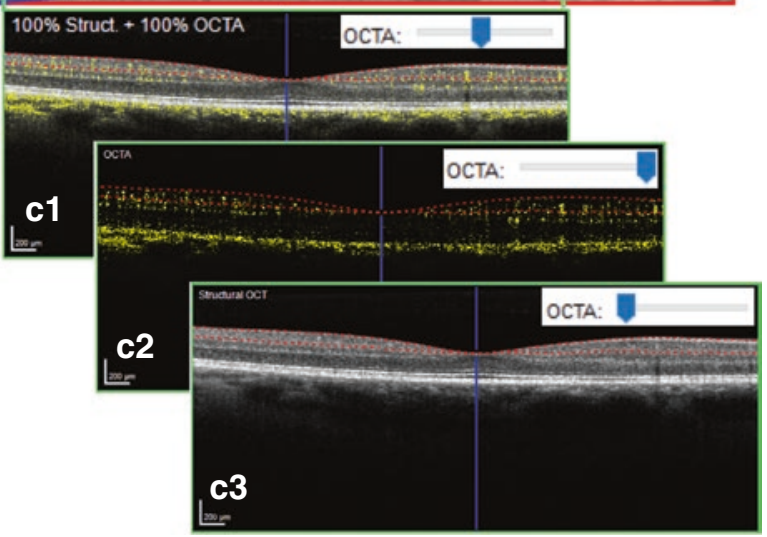

segmentations of the structural OCT data (see Sect. 6.2.4). The OCTA signal between the two boundaries is accumulated in axial direction using different projection methods (see Sect. 6.2.3) and is displayed as a two-dimensional image. The resulting images give the impression of looking onto the retina and are therefore referred to as en face images or transverse section images. An example is given in Fig. 6.2a, showing the en face image of the structural OCT, and Fig. 6.2b, showing the respective en face image of the OCTA signal.

In addition to en face images, section images are used for review of the spatial relationship of retinal structure and blood flow. The section 
images may have the same orientation as the originally acquired B-Scans (Fig. 6.2c) but may also be arbitrarily oriented within the volume; for instance Fig. 6.2d shows a section orthogonal to the original B-Scans. To provide a direct visual correlation of structural and flow information, structural OCT section images and the corresponding OCTA blood flow information at the same location can be superimposed; see Fig. 6.2c1-c3, d.

\subsubsection{Projection Methods}

The generation of two-dimensional en face images from the three-dimensional data, $\operatorname{OCTA}(x, y, z)$, employs a projection along the $z$-direction (i.e. axial direction). Common projection methods are the mean projection, $\mu(x, y)$, and the sum projection, $s(x, y)$, which can be discretized at voxel-level and written as

$$
\begin{gathered}
\mu(x, y)=\frac{1}{z_{l}(x, y)-z_{u}(x, y)} \sum_{z=z_{l}(x, y)}^{z_{u}(x, y)-1} \operatorname{OCTA}(x, y, z(x, y)) \\
s(x, y)=\sum_{z=z_{l}(x, y)}^{z_{u}(x, y)-1} \operatorname{OCTA}(x, y, z(x, y))
\end{gathered}
$$

where $z_{l}(x, y)$ is the posterior slab boundary surface, and $z_{u}(x, y)$ is the anterior slab boundary surface, so that the slab comprises all data with $z \in\left(z_{u}, z_{l}\right] \subset Z$. Note, the only difference between these two projection methods is the normalization factor. The mean projection is normalized by the local slab thickness, $d(x, y) \equiv z_{l}(x, y)-z_{u}(x, y)$, while the sum projection is not normalized. This has important implications for the visual interpretation of the resulting en face images and also for the use of these images in OCTA analytics. To appreciate the differences, assume that the OCTA algorithm achieves a perfect result without any artifacts or noise, i.e. $\operatorname{OCTA}(x, y, z)$ is 1 for any voxel corresponding to a perfused retinal location and 0 for any voxel corresponding to a nonperfused location. In this idealized setting $\mu(x, z)$ can be interpreted as a density or fill-factor of perfused vessels in the slab, i.e. the ratio of perfused voxels to all voxels in the depth range from $z_{l}(x, y)$ to $z_{u}(x, y)$. The sum projection, $s(x, y)$, corresponds to the total lumen of perfused vessels in the given depth range. Both measures can be meaningful, depending on how the images are read or further processed. The density of perfused vessels in a given slab may give direct insight into the relative oxygenation of this slab. But due to laterally varying slab thickness, $d(x, y)$, the contribution of small capillaries in the mean projection also laterally varies within the same slab. This is illustrated in Fig. 6.3. The contribution to the mean projection is greater if a capillary is located somewhere with small slab thickness (Fig. 6.3b) as compared to a same sized capillary at a location of greater slab thickness (Fig. 6.3c). Considering that the slab thickness might also vary over time (due to swelling of the retina or treatment of fluid accumulations), the contribution of capillaries in the mean projection also changes over time, even if the capillary's perfusion is unchanged. In contrast, the sum projection always gives equal weight for each voxel, independent on the local slab thickness. This means that all capillaries of a given diameter contribute equally to the sum projection. On the other hand, using the sum projection for slabs with laterally varying thickness may give the false impression of non-perfusion at locations where the slab is thin and only comprises single capillaries, when compared to a location where the slab is thick enough to embrace more than one layer of capillaries. To avoid this false impression, it is important to realize that the sum projection is related to the perfused vessel lumen at each lateral location, which is naturally confined by the local slab thickness.

The previous examples were given to illustrate the difference when reading the en face images. For OCTA analytics based on en face images there are further aspects that need to be 

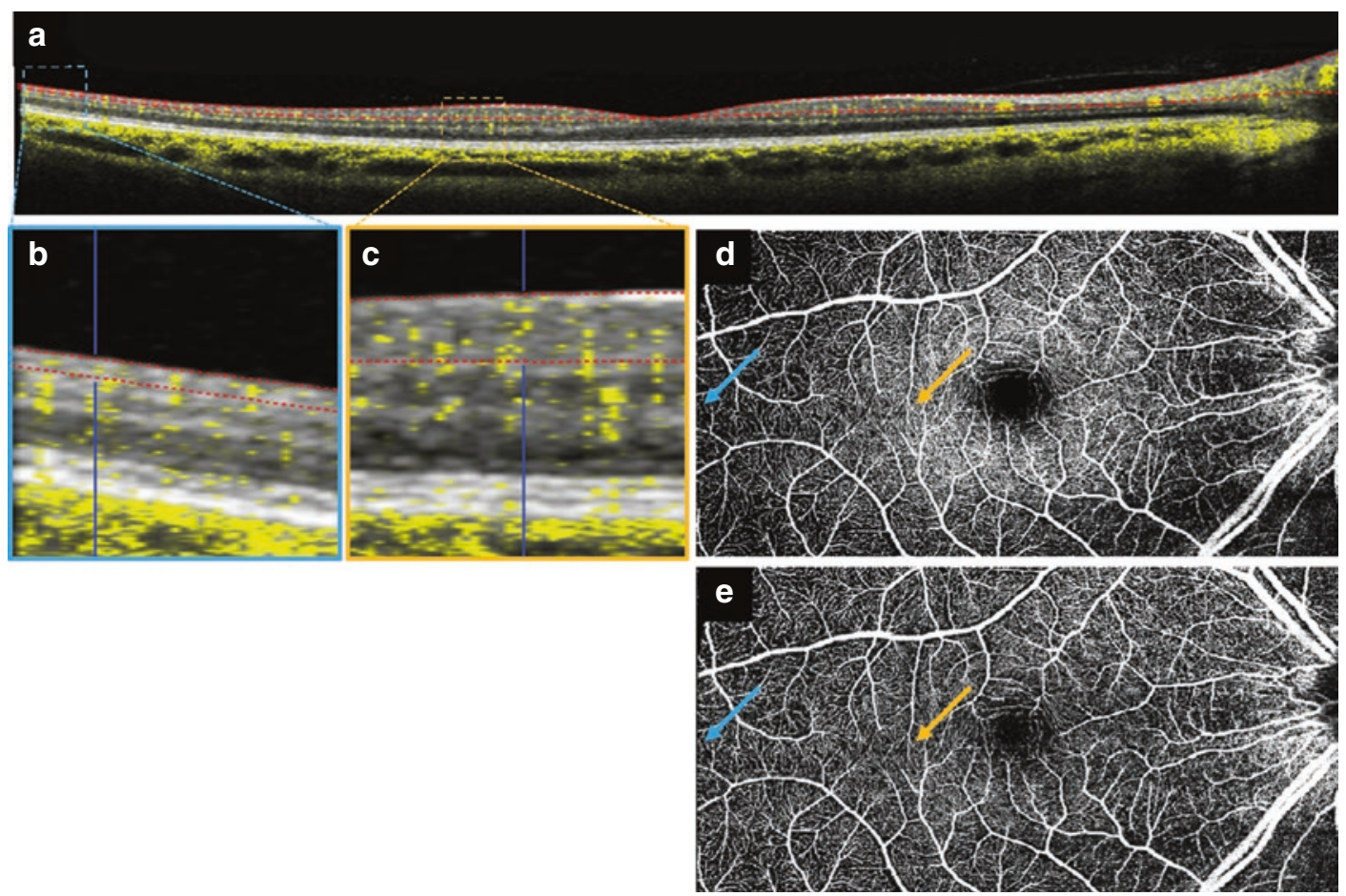

Fig. 6.3 Comparison of OCTA sum and mean projection. (a) Fusion image with SVC slab boundary segmentation (red lines). (b, c) Detailed fusion images show the physiological thinning of the superficial vascular plexus from

considered. When using the mean projection as a measure for vessel density within several slabs of varying thickness, it is not possible to directly compare the results because voxels were given different weights in the different slabs. Also averaging of mean projections from different slabs will not give the same result as the computation of the mean projection of the combined slab.

\subsubsection{Retinal Vascular Plexuses}

The retinal vascular network in the human eye is axially divided into four distinct capillary plexuses. While the vasculature within each plexus is densely linked, interconnecting vessels between these sub-networks are sparse in comparison. Each separate capillary plexus holds characteristic morphometric features that have been confirmed ex-vivo in confocal microscopy $[3,12]$ as well as by using OCTA in vivo $[13,14]$. From the the macula to the periphery. (d) Sum projection of the SVC slab. (e) Mean projection of the same slab. Blue and orange arrows show the location of (b) and (c), respectively

anterior boundary of the retina to more posterior axial locations, the four distinct plexuses are, nerve fiber layer vascular plexus (NFLVP), superficial vascular plexus (SVP), intermediate capillary plexus (ICP), and deep capillary plexus (DCP); compare Fig. 6.4.

While the three deeper layers can be observed and axially separated even in the periphery of the retina using OCTA at sufficient axial resolution, the NFLVP is most pronounced at locations where the nerve fiber layer holds a substantial width, like in the peripapillary region as well as parafoveally (Fig. 6.5) [14].

In order to accurately detect and manage retinal vascular conditions, it is important to precisely discern the different retinal vascular plexuses. It is also important that slabs enable a continuous representation of the retinal and choroidal vasculature so that possible vascular abnormalities are not missed during image review. Currently, conflicting definitions of the axial location of bound- 


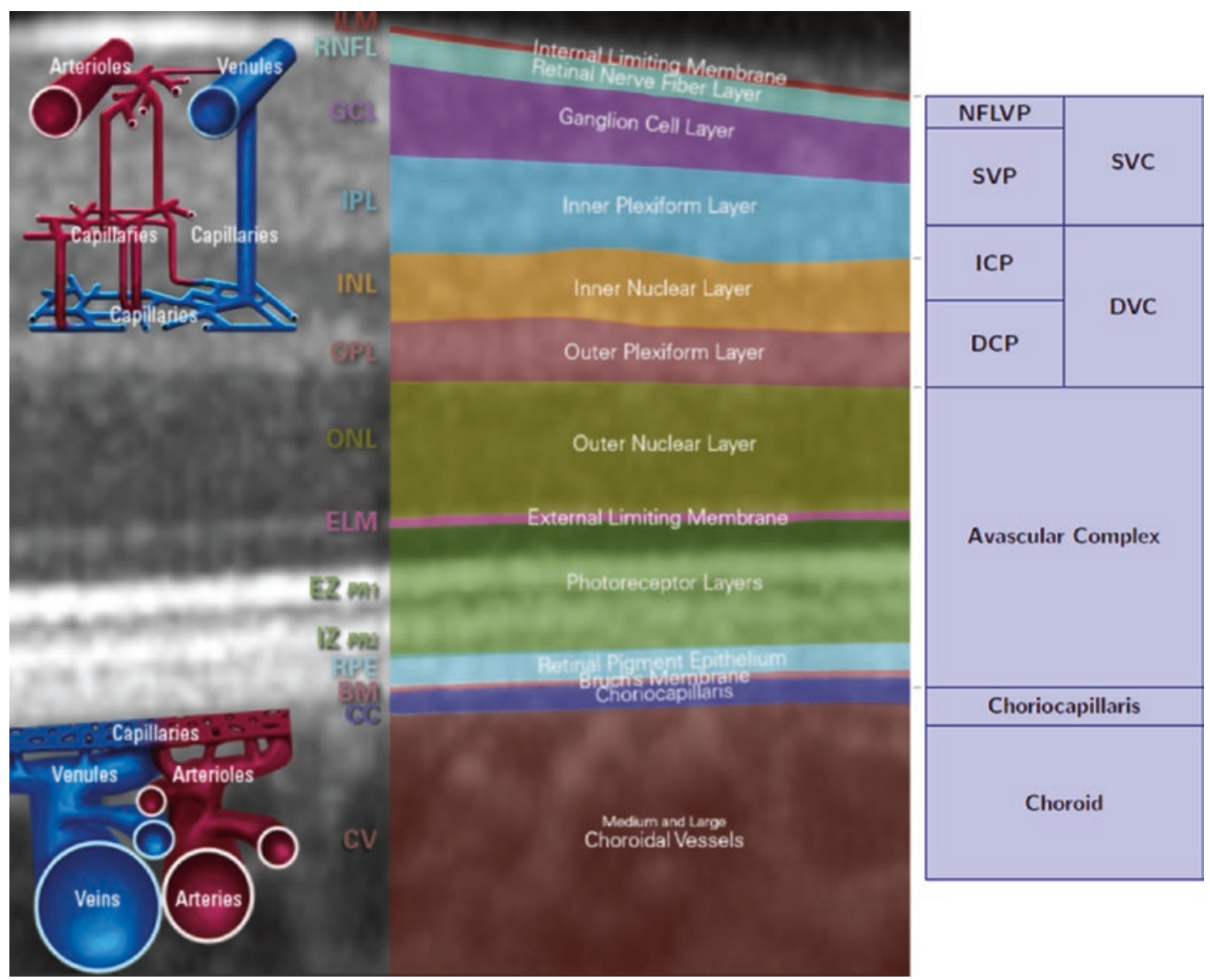

Fig. 6.4 Definition of the slab boundaries. Left: Schematic figure of the layers and vessel networks in the human retina (www.he-academy.com/Retinal-LayersInteractive). Right: Schematic figure of the slab definitions. SVC superficial vascular complex, NFLVP nerve fiber layer vascular plexus (part of SVC), SVP superficial vascular plexus (part of SVC), DVC deep vascular complex, $A C$ avascular complex, $I C P$ intermediate capillary

aries between retinal plexuses make the direct comparison of en face images from different devices difficult [16].

To best separate distinct capillary plexuses within the deep vascular complex, Campbell et al. suggested to define optimum slab boundaries based on the location of minima of the axial flow density profiles [13]. They introduced boundary definitions relative to the thickness of the segmented retinal layer. As an alternative to this approach, a subsequent study using full spectrum OCTA at higher axial resolution found that it is also possible to define these interfaces plexus (part of DVC), $D C P$ deep capillary plexus (part of DVC), $C C$ choriocapillaris. Figure modified from https:// www.heidelbergengineering.com/download.php?https:// media.heidelbergengineering.com/uploads/ProductsDownloads/210111-001_SPECTRALIS_Tutorial_ SPECTRALIS-OCTA-Principles-and-ClinicalApplications_EN.pdf [16]

between the three deeper layers at constant absolute offsets to the retinal IPL-INL interface (Fig. 6.5) [14]. This conveniently reduces the number of required retinal segmentations that are necessary for creating individual en face visualizations of these plexuses (cf. Fig. 6.6c, d).

\subsubsection{Quantification of OCTA Data}

For objective assessment of disease progression and its documentation, and to enable comparisons to normative data, a concise summary of the 

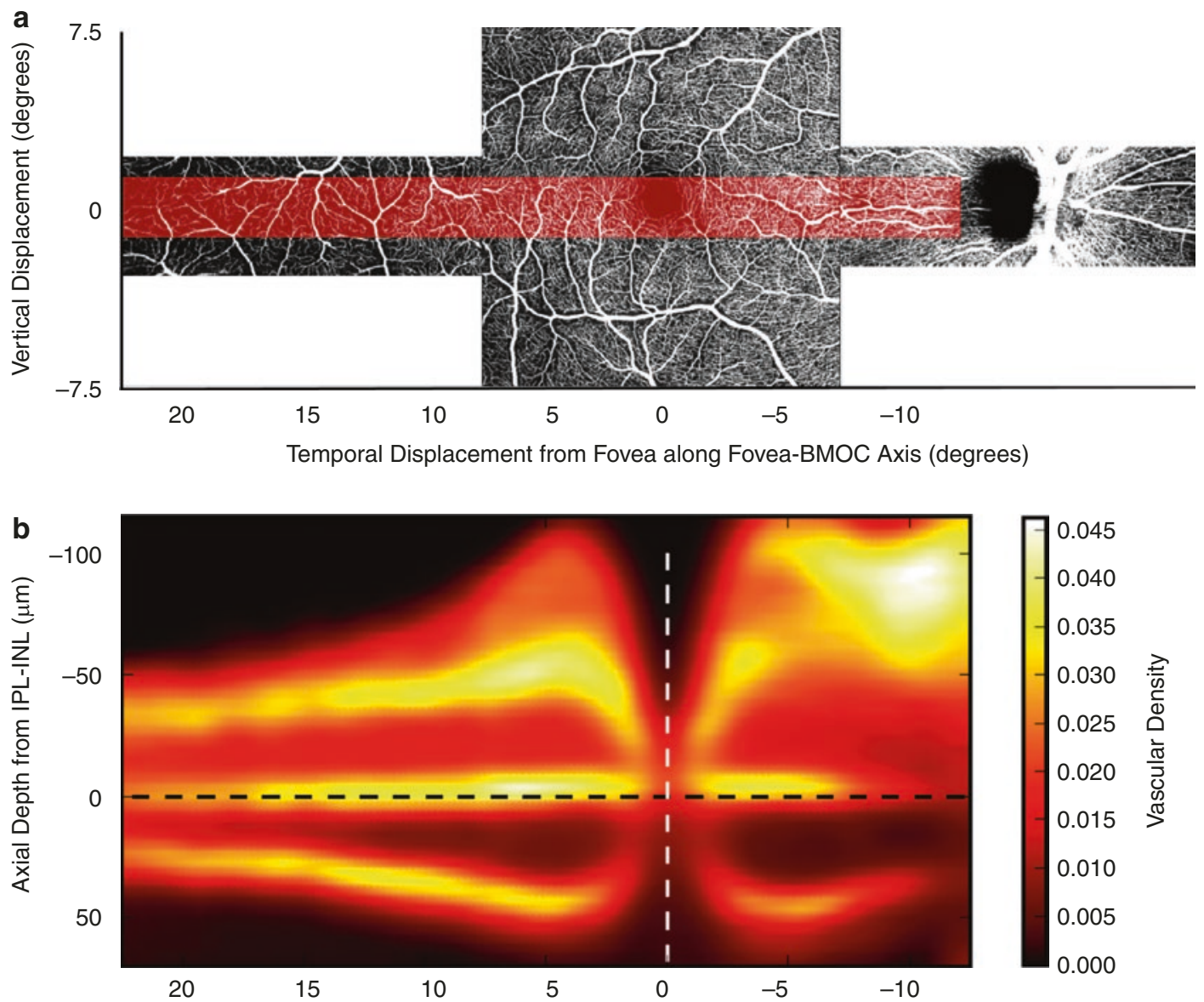

Temporal Displacement from Fovea along Fovea-BMOC Axis (degrees)

Fig. 6.5 (a) Representative en face OCTA image of the superficial retinal vasculature from the optic nerve head across the fovea to the temporal periphery in a healthy eye. (b) Heat map of the OCTA signal in depth averaged over 22 healthy eyes, spatially averaged within the transparent red overlay displayed in (a). Hot locations (white) indicate strongest OCTA signal while cold locations (black) indicate minimal OCTA signal. Up to four axially distinct capillary plexuses can be detected in the peripap- illary region as well as parafoveally. At peripheral temporal locations, three distinct plexuses are separated. The retinal layer interface between IPL and INL, when shifted anteriorly and posteriorly by constant appropriate distances, represents a conveniently defined separating boundary for visualizing the three deeper plexuses independently within en face projections. Image from [14] reproduced without changes according to license http:// creativecommons.org/licenses/by/4.0/ image data in terms of numerical measurements is desirable. Such numeric parameters, describing the structure of the vasculature network as derived from the OCTA images, are also referred to as "OCTA metrics" or "OCTA analytics". Clearly, this must not be confused with OCT-based flowmetry (subject of Chap. 7), where physical blood flow velocity is measured quantitatively.

Typical examples of OCTA metrics parameters, quantifying static structural aspects of the eye's vasculature, are various vessel density measures (vessel/perfusion density, binarized vessel density or vessel area density, skeletonized vessel density or vessel length index). These parameters are suitable for capturing dropout of vasculature that occurs in diseases like diabetic retinopathy, retinal vein occlusion or glaucoma [17-20]. A quantification of flow void area is also possible, for instance for assessing the choriocapillaris structure [21]. 


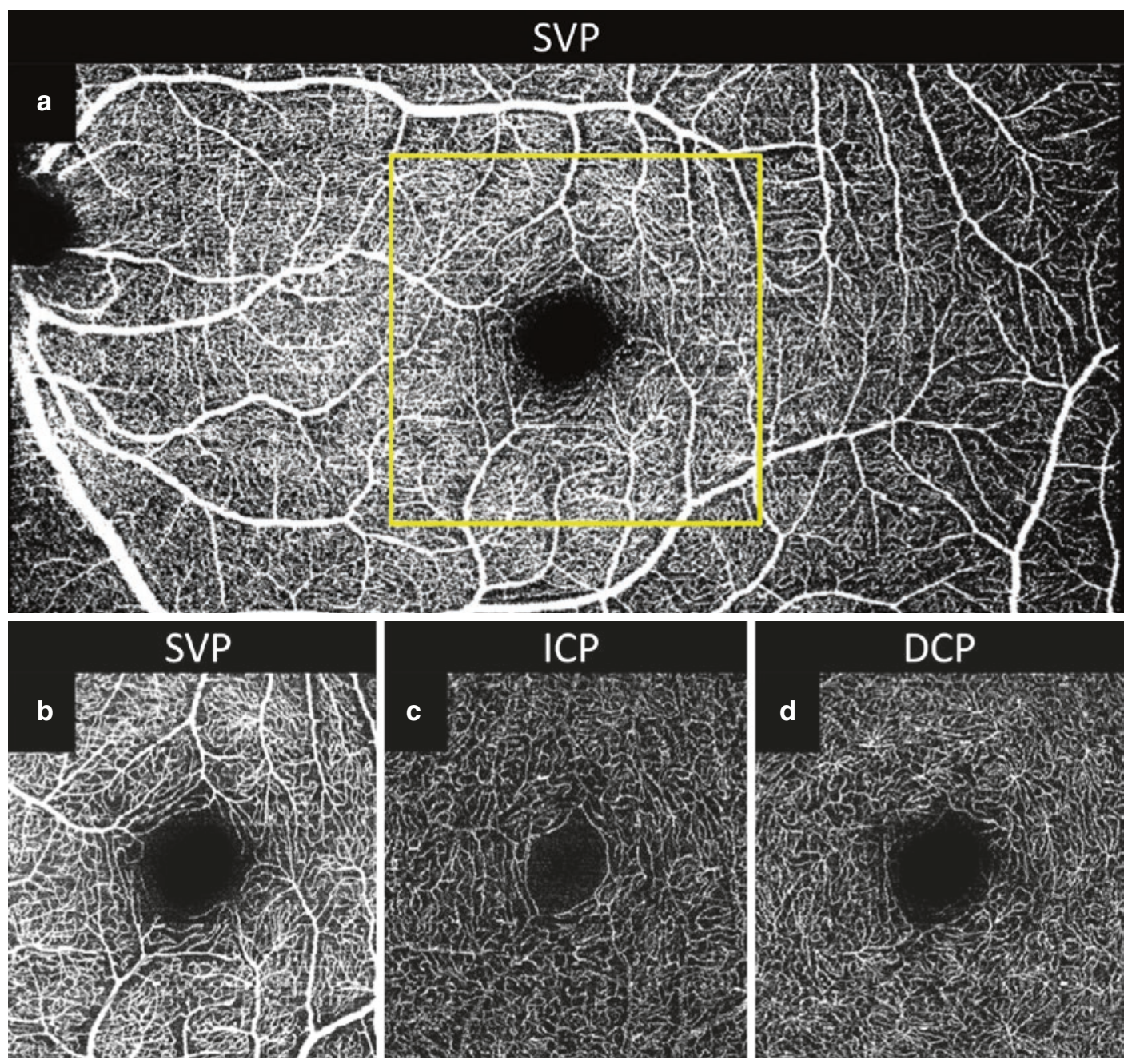

Fig. 6.6 Comparison of two examinations of the same eye. (a) En face image of the superficial vascular plexus (SVP) from $30^{\circ} \times 15^{\circ}$ scan acquired with resolution of $11 \mu \mathrm{m} /$ pixel, providing a large field of view. (b-d) En face images acquired of a $10^{\circ} \times 10^{\circ}$ scan with $5.7 \mu \mathrm{m} /$ pixel resolution. (b) The small capillaries are better resolved in the SVP en face image of high resolution scan, compare yellow outline in A which shows the same region of the

Besides vessel density, other parameters that summarize morphological features of vessel branches and vessel network structure, including complexity measures such as vessel tortuosity, fractal dimension, or branching point densities and vessel diameter statistics, are in common use [17$19,22,23]$. These measures aim at capturing pathological alterations of vessel shape and spatial arrangement, as occurring for example in diabetic same eye. (c) The intermediate capillary plexus (ICP) can be clearly distinguished from the deep capillary plexus (d) due to the high axial resolution of $\sim 3.9 \mu \mathrm{m} / \mathrm{pixel}$ (SPECTRALIS OCTA). The ICP and DCP vessel networks show clearly distinct geometric structures. In the DCP, star-like vascular intersections can be discerned which may represent a connection to the venous superficial network

retinopathy, macular telangiectasia, or neovascularization in age related macular degeneration.

These characteristics may be analyzed for the whole scan area, or alternatively as aggregates over sectors defined by specific grids (e.g. ETDRS grids), which are usually adapted to the eye anatomy and allow for the detection of spatially localized changes over time or deviations from the statistics of normal reference data. 
In currently available approaches, vessel density measures are interpreted as a two-dimensional density, i.e. the fraction of area of a slab projection occupied by detected vessels (typically after applying a thresholding operation). As long as the instrument is able to axially resolve the different layers of capillaries that can be anatomically distinguished, quantitative OCTA parameters can be derived for each of them independently. To suppress the influence of larger vessels, two approaches are commonly used: Either slab projections of vessels are reduced to their centerlines (i.e. "skeletonization"), or larger vessels are simply masked out. This emphasizes thinner capillaries in the analysis.

Further quantitative parameters derived from OCTA data are area and shape measurements of specific vasculature regions, in particular the foveal avascular zone (FAZ) [19, 22] or segmented neovascular lesions [24].

There is high interest to use results from quantitative OCTA parameters as endpoints in clinical studies [25-27]. For this purpose, OCTA metrics need to be both repeatable and reproducible. Therefore, initial studies mostly focused on analyzing the robustness of the measurements. Errors in scan geometry, evaluation grid placement, layer segmentation, and parameters such as variable signal strength can negatively impact the measurement precision. Furthermore, data from instruments of different vendors are not directly comparable [28, 29]. This is due to differences in resolution as well as signal generation and postprocessing algorithms such as filtering, artifact suppression and thresholding [30]. Also differences in the slab definitions (cf. Sect. 6.2.4) and layer segmentation results of different devices need to be carefully taken into account when comparing images or quantitative analysis results across devices.

\subsection{Image Artifacts and Countermeasures}

\subsubsection{Projection Artifacts}

The OCT light passage through larger superficial vessels can introduce disturbances to the probing beam in deeper retinal layers. The light fluctuates because it has passed through and is altered by moving blood cells above. Current OCTA algorithms cannot distinguish these signal fluctuations from the fluctuations of moving blood cells in the deeper layer. This gives rise to apparent replications of superficial vessels in posterior layers. These erroneous replications are referred to as OCTA projection artifacts [5]. Projection artifacts are introduced during data acquisition and are not a consequence of the projection method for en face image generation. While projection artifacts were understood as the most confounding factor early on in the inception of OCTA technology $[5,7]$, this limitation has been addressed in current state-of-the-art devices by means of a post-processing step which is referred to as projection artifact removal [4, 31, 32]. Typically, in this step either the undesirable replication is subtracted from en face projections of the deeper layer by algorithmic comparison to its source in the superficial vasculature or the deeper axial signal is suppressed at selected locations based on heuristic rules [32]. In general, projection artifacts are more prominent in deeper layers of high signal intensity, such as the retinal pigment epithelium (RPE). Therefore, it is important that the projection artifact removal does not lead to disruption of the visualization of structures such as choroidal neovascularization (CNV) which may grow through the RPE. For example in Fig. 6.7a, various large vessels seem to be connected to a large CNV and the actual extent cannot be easily assessed. With projection artifact removal, Fig. 6.7b, the artificial replication of the vessels from superficial layers can be removed without disrupting the visualization of the pathology.

\subsubsection{Segmentation Artifacts}

Considering that slabs are mainly defined by automatically segmented retinal layer boundaries, careful review of the segmentation is critical for correct interpretation of the en face projections. Segmentation failures are especially common in diseases where the appearance and shape of retinal layer is altered. For instance, intrareti- 


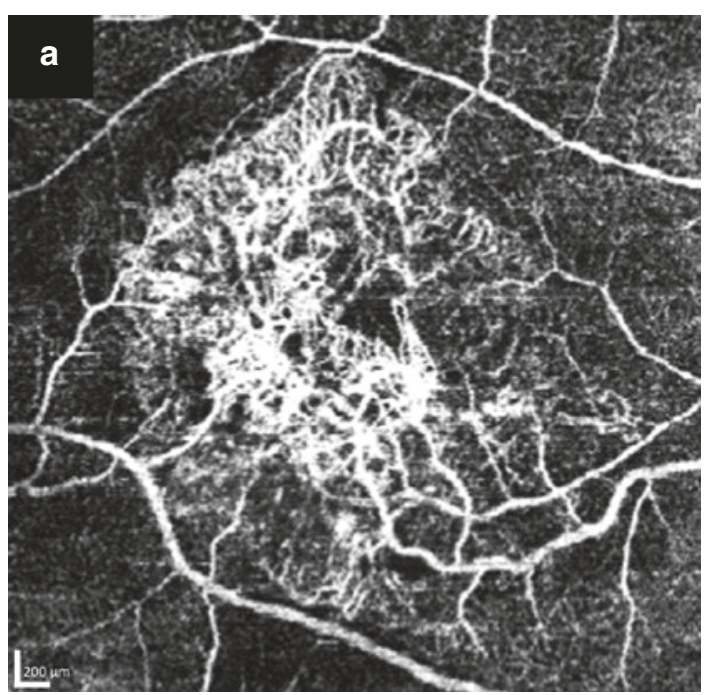

Fig. 6.7 A subject with a large type $1 \mathrm{CNV}$ was examined with $11.4 \mu \mathrm{m} /$ pixel resolution using SPECTRALIS OCTA. (a) OCTA image without projection artifact removal, various large vessels seem to be connected with

nal fluid, large pigment epithelial detachments, choroidal neovascularization, and certain atrophies often cause segmentation errors in state-ofthe-art OCTA devices. A manual correction of such errors is cumbersome, if the correction is based on individual B-Scans within the dense volume. To facilitate and speed up the process of correcting compromised slab boundaries, interactive segmentation correction tools have been introduced. These tools propagate manual corrections of only a few B-Scans to the remainder of the volume, by fusing automatic segmentation results with these user-provided hints [33]. These countermeasures are merely a workaround until more robust segmentation methods for the vascular networks are available.

\subsubsection{Motion Artifacts}

Adequate compensation of eye motion is one of the most critical aspects of OCTA acquisition. In order to detect temporal changes in the OCT signal related to blood flow in capillaries, eye motion needs to be detected and compensated for very accurately. Therefore sampling schemes and different eye tracking implementations play a cru-

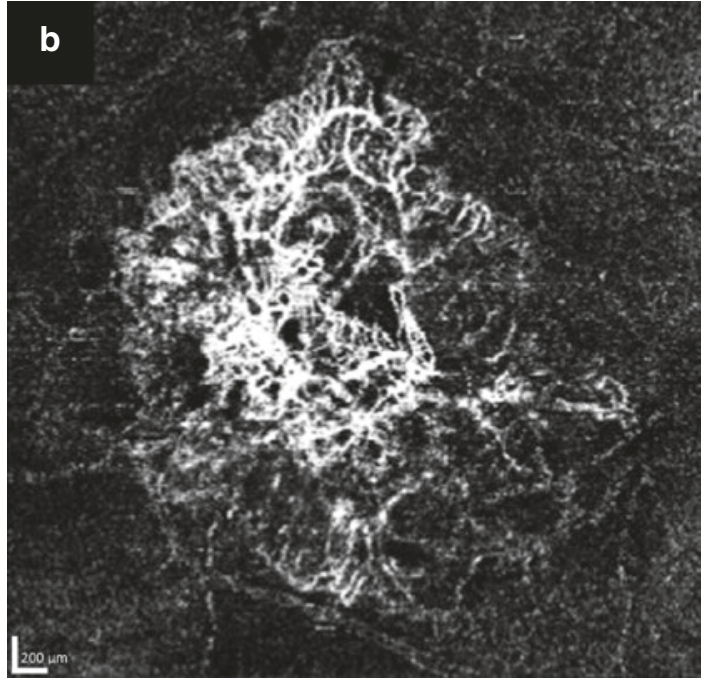

the neovascularization. (b) After removal of the projection artifact, the actual size and the extent of the neovascularization can be assessed

cial role for each device's overall performance of blood flow visualization at the capillary level.

Eye movements affect the acquisition of OCTA data in two distinct ways. First of all, the detection of blood flow related changes in the OCT signal from repeated B-Scans requires spatial overlap of these scans (see Sect. 6.2.1). This means, that the scans must overlap within the lateral width of the probing beam, which is typically in the order of $15 \mu \mathrm{m}$. Secondly, larger eye movements can lead to geometrical distortion or missing data in the OCTA volume scans. The volume scans are typically acquired within several seconds. On this time scale, it is very likely that larger eye movement occurs due to saccades, change of fixation, or change of head pose.

Slow eye drifts within the B-Scan plane can be compensated for by image registration of the successive B-Scans, effectively removing the temporal signal change in regions of static tissue (cf. Fig. 6.1). Larger eye motion (saccades) or eye motion perpendicular to the B-Scan plane can strongly deteriorate the OCTA signal, because of insufficient spatial overlap of the B-Scan samples. This leads to missing data and geometrical distortions in the OCTA volume scans, if no countermeasures are taken. The goal 
of any motion artifact compensation is to ensure that OCTA data acquired from visit to visit is devoid of errors that may reduce the precision of quantitative change analyses.

There are two major approaches to mitigate motion artifacts in OCTA. One approach is to acquire several independent OCTA volume scans and combine the information in post-processing [34-36]. The second approach is using real-time tracking.

The post-processing approach has the advantage of relatively short acquisition times. In practice, often only two volume scans with perpendicular orientation of the fast scanning axis are used [36]. In effect, the resulting volume is obtained by interpolation and averaging of the different input volumes. Larger data gaps from one volume are normally filled up with information from the other volume, but in general, there is no guarantee to obtain distortion-free results [5].

The real-time tracking approach employs accurate measurements of the eye motion in realtime (see also Chap. 3). B-Scans which are affected by too strong motion are re-acquired. During periods of slow eye motion (eye drifts) the real-time eye motion measurements can also be used to actively control the OCT scanners to keep the beam on the nominal scanning path. Due to the data filtration in the event of strong eye motion, the real-time tracking approach is sometimes slow in acquisition. However, using realtime tracking, the obtained volumes are geometrically accurate and uniformly sampled without gaps from missing data.

\subsubsection{Lateral and Axial Resolution}

The lateral resolution of an OCT system is determined by both the optical point spread function (PSF) as well as sampling density, i.e. the digital resolution. The OCTA signal from one voxel can be seen as a mixture of contributions from scatterers within the support of the combined (optical and digital) PSF. The larger the PSF, the more scatterers contribute to the mixture so that is becomes more challenging to separate the individual contributions, in particular the compo- nents due to flowing scatterers (blood cells) from the static components (tissue) according to their discriminating statistics.

The optical PSF is influenced by the imaging system as well as the imaged eye. Poor adjustment of the instrument's focus as well as aberrations in the eye widens the PSF and lead to suboptimal signal separation. Similarly, using wide-field optics with smaller numerical aperture as well as less dense digital sampling in favor of covering larger fields of view compromises the ability to resolve small capillary details. The effect of the sampling density on the visibility of small capillaries is illustrated in Fig. 6.6, compare $a$ and $b$.

The axial resolution in OCT is independent of the lateral resolution and is determined by the spectral bandwidth of the light source [37]. However, using split-spectrum approaches [34] for OCTA processing, an algorithmic trade-off can be made between axial resolution and signal to noise ratio. Splitting the spectrum allows to obtain, from a single scan, several B-Scan sections of lower axial resolution (due to the lower bandwidth of the spectral sub-bands), which are then used as additional samples with independent shot noise contributions for improving the OCTA signal. This loss of resolution may impede the ability to separate axially closely spaced but distinct vascular layers, in comparison to algorithms that maintain the axially high optical resolution of the underlying OCT signals (cf. Fig. 6.6c, d) [14].

\subsection{Clinical Application of OCTA}

\subsubsection{Diabetic Retinopathy}

Diabetic retinopathy (DR) is classified into different stages according to stereoscopic color fundus photographs. The typical features of DR are microaneurysms, intraretinal hemorrhages, intraretinal microvascular abnormalities, venous beading, cotton wool spots, hard exudates and neovascularization.

The gold standard imaging technique for the assessment of macular perfusion is fluorescein angiography [38]. It is able to show leaking 
microaneurysms and capillary non-perfusion. However, it produces only two-dimensional images in which fluorescence signals of the superficial and deep capillary networks overlap and are difficult to distinguish, especially when the dye leaks $[38,39]$. In this context, OCTA is a useful imaging technique for the assessment of retinal vasculature in the different capillary networks.

Fluorescein angiography is a dynamic examination that can be used to accurately evaluate the perfusion status of the retina at the posterior pole and in the far periphery. In contrast, the application of OCTA in the assessment of peripheral vasculature is quite controversial. In fact, one of the main deficiencies of this imaging technique is related to its limited field of view suggesting a use for diseases affecting the macular region. However, several features of DR have the potential to be imaged and more accurately classified with OCTA as it offers the opportunity to show various retinal vascular layers [40, 41]. In fact, OCTA is currently being included into clinical trials of patients with DR. There may be prospects to describe the evolution of the DR as OCTA offers the opportunity to evaluate the quantification of perfusion through vascular density maps, and the potential for feature identification such as identifying microaneurysms or specified regions of non-perfusion (Figs. 6.8, 6.9, and 6.10) $[7,17]$.

\subsubsection{Retinal Vein Occlusion}

Retinal vein occlusion is the second most prevalent blinding vascular retinal disorder [42, 43]. The main types are central retinal vein occlusion (CRVO) and branch retinal vein occlusion (BRVO), depending on the site of obstruction [44]. Examples are shown in Figs. 6.11 and 6.12.

The ophthalmoscopic signs are venous dilation, tortuosity, intraretinal hemorrhage, retinal edema and hemorrhage in the vicinity of the occluded vein. Fluorescein angiography typically reveals delayed filling in the distribution of the involved retinal vessels. The veins are dilated and tortuous. There is leakage from capillaries with dye accumulating in the substance of the retina or within cystoid spaces. Fluorescein angiography has been the gold standard for visualizing retinal non-perfusion areas which appear as dark areas in the images [38]. Retinal neovascularization can be identified by leakage of the fluorescein dye. Fluorescein angiography has been critically important for the diagnosis and the treatment decision.

OCTA shows the areas of vascular nonperfusion, the dilated tortuous venous segments, the microvascular abnormalities and the neovascularization. However, one of the most sight threatening complications is macular edema. In this context, it is crucial to verify the segmentations used for OCTA visualization [45]. The difficulty with en face imaging is that selective enlargement of retinal layers occurs and these layers are typically not segmented correctly. The resultant en face vascular images may not show the correct representations of the actual flow characteristics.

\subsubsection{Macular Telangiectasia}

Idiopathic perifoveal or juxtafoveolar retinal telangiectasia are retinal capillary ectasia limited to the perifoveal area without any apparent specific cause [46].

Initially, idiopathic macular telangiectasia were divided into four groups according to the Gass classification [47]. Subsequently, Yannuzzi proposed a new classification in which type 1 was defined as aneurysmal telangiectasia and type 2 as perifoveal idiopathic macular telangiectasia [48].

Type 1 is closely related to Coats disease, or more specifically a milder form of Coats previously known as Leber miliary aneurysms. It generally involves only one eye, and both the peripheral retina and macula can be affected. Fluorescein angiography reveals telangiectasia and multiple capillary, venular and arteriolar aneurysms with late leakage. Type 2 are bilateral, temporal and symmetrical, however, there have been reports of unilateral, asymmetric, and asymptomatic cases [49]. It has been hypothe- 

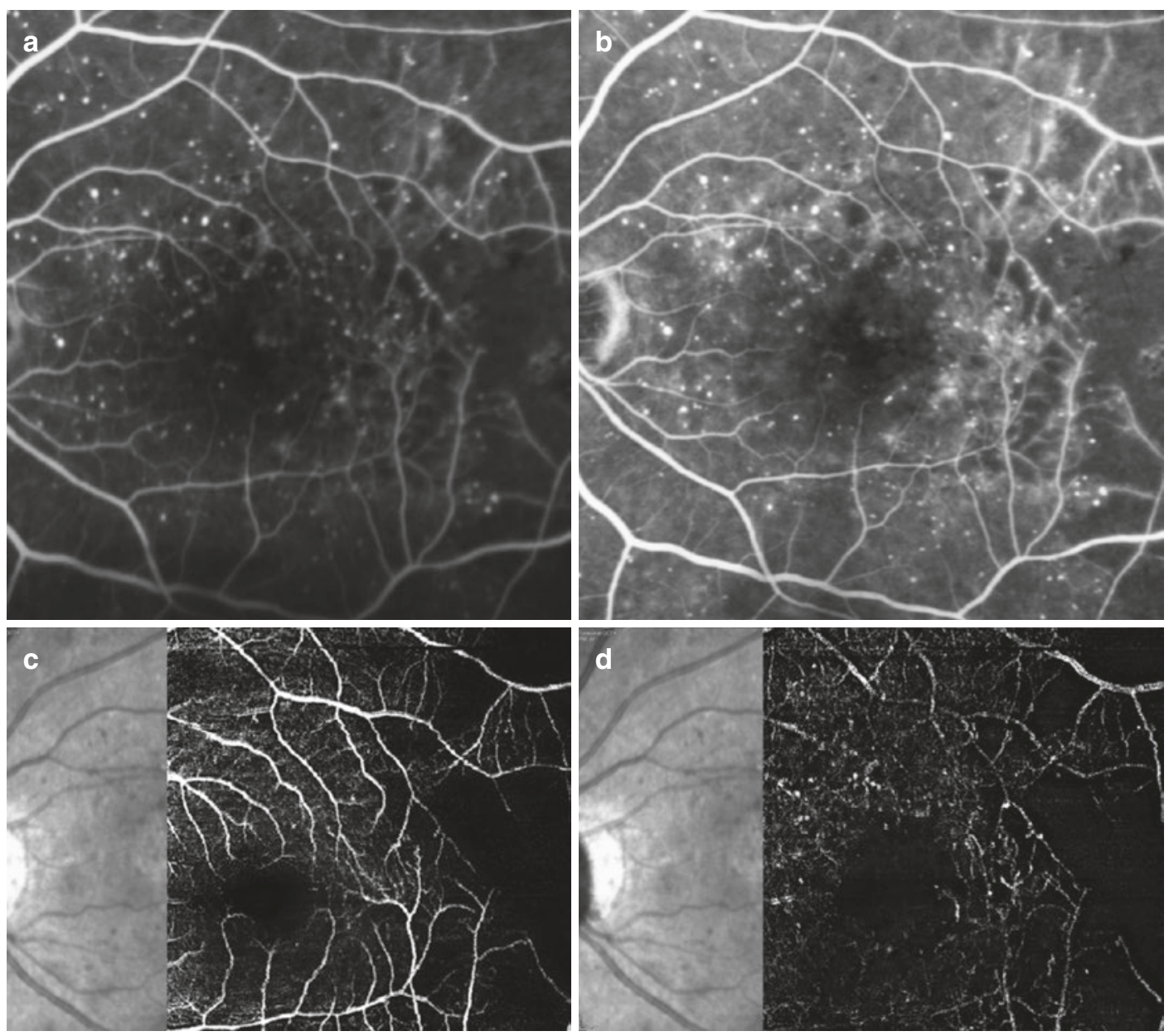

Fig. 6.8 Multimodal imaging of diabetic retinopathy. Fluorescein angiography (a, b) and the corresponding optical coherence tomography angiography images segmented at the superficial retinal capillary plexus (c) and the deep capillary plexus (d). Fluorescein angiography

revealing vascular changes with microaneurysms and an area of non-perfusion in the temporal part. Optical coherence tomography angiography showing microaneurysms and a similar aspect of the area of non-perfusion

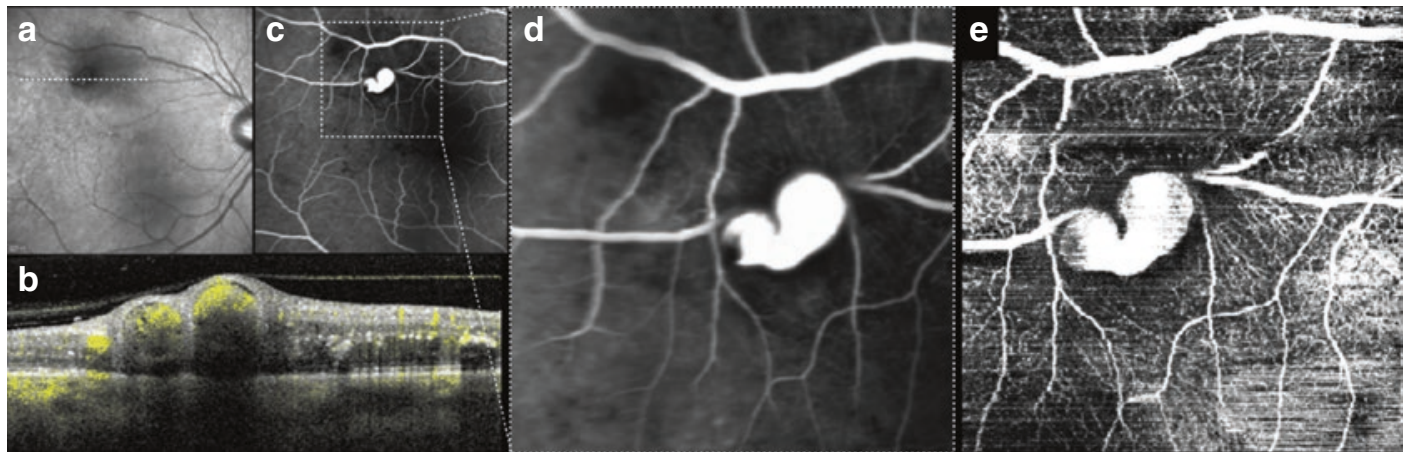

Fig. 6.9 Multimodal imaging of macroaneurysm. Infrared reflectance (a) showing fundus abnormalities on a temporal vascular arcade corresponding to vascular dila- tion on OCT (b). Fluorescein angiography $(\mathbf{c}, \mathbf{d})$ and OCTA (e) revealing the macroaneurysm with a similar representation 

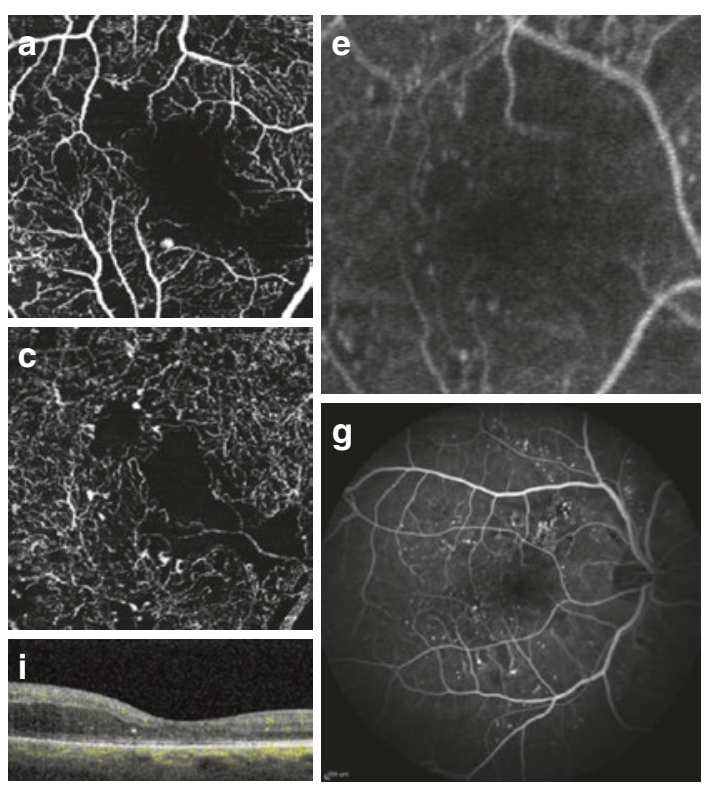

Fig. 6.10 Multimodal imaging of diabetic retinopathy. OCTA images segmented at the superficial retinal capillary plexus $(\mathbf{a}, \mathbf{b})$ and the deep capillary plexus $(\mathbf{c}, \mathbf{d})$, the corresponding fluorescein angiography in the early $(\mathbf{e}, \mathbf{f})$ and more advanced phase $(\mathbf{g}, \mathbf{h})$ and the corresponding
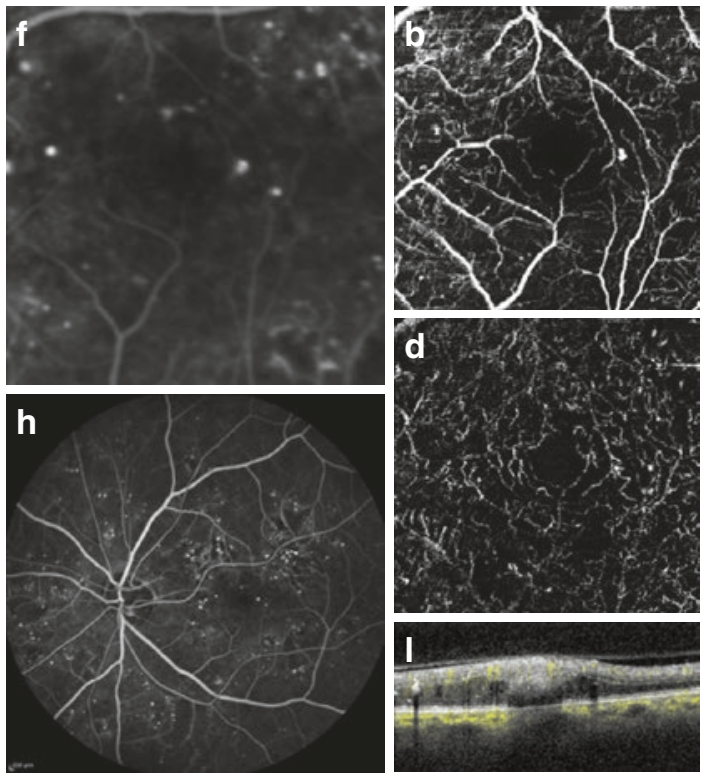

B-Scan (i, j). OCTA showing microvascular changes, microaneurysms, vascular dropout and a ragged appearance of the foveal avascular zone. Some microaneurysms are seen on the fluorescein angiography but not on the OCTA images

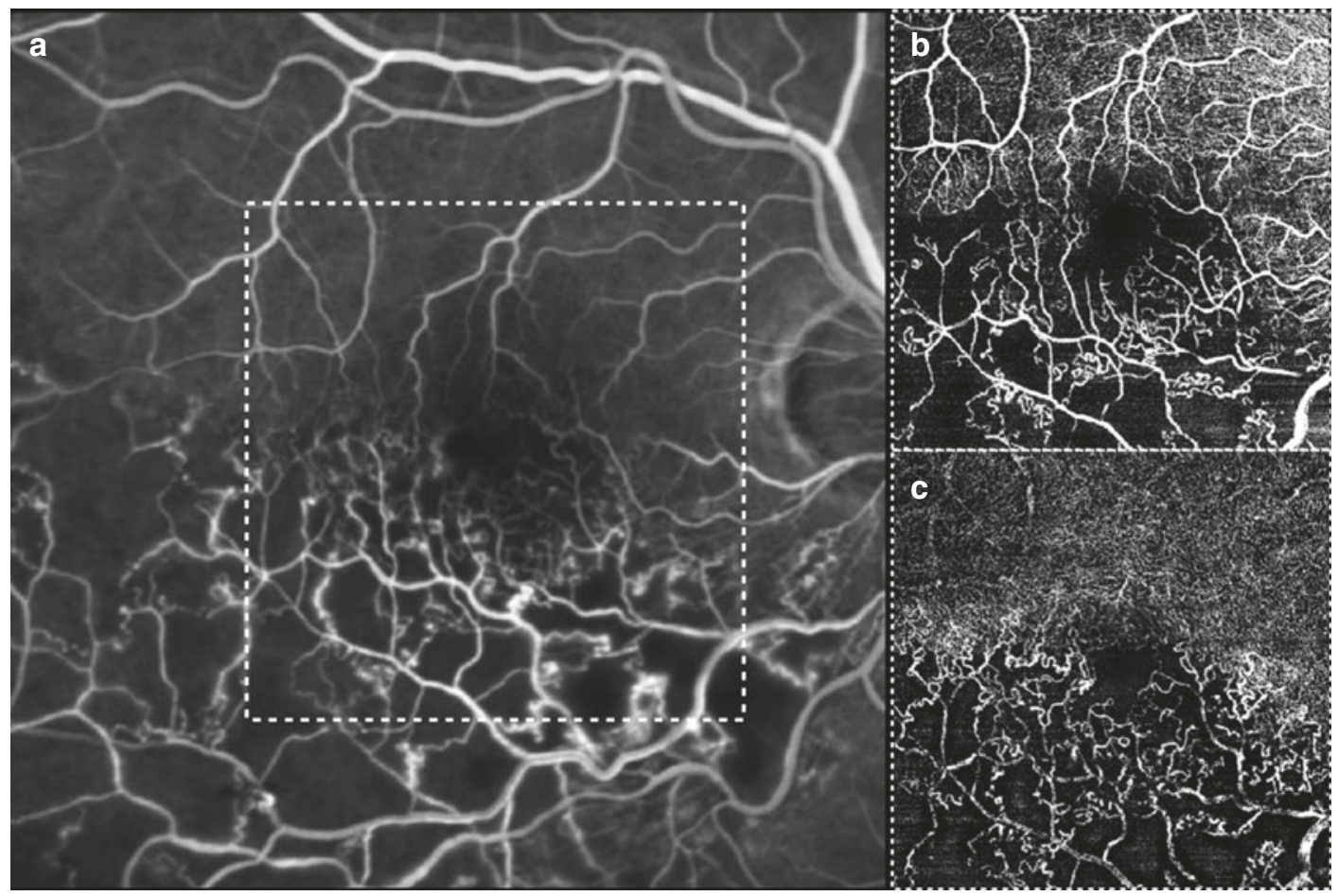

Fig. 6.11 Multimodal imaging of retinal vein occlusion. Fluorescein angiography (a) and the corresponding optical coherence tomography angiography images segmented at the superficial retinal capillary plexus (b) and the deep capillary plexus (c). Fluorescein angiography revealing vascular changes and area of non-perfusion in the temporal part of the retina. Optical coherence tomography angiography showing well the area of vascular nonperfusion with a similar appearance of fluorescein angiography 

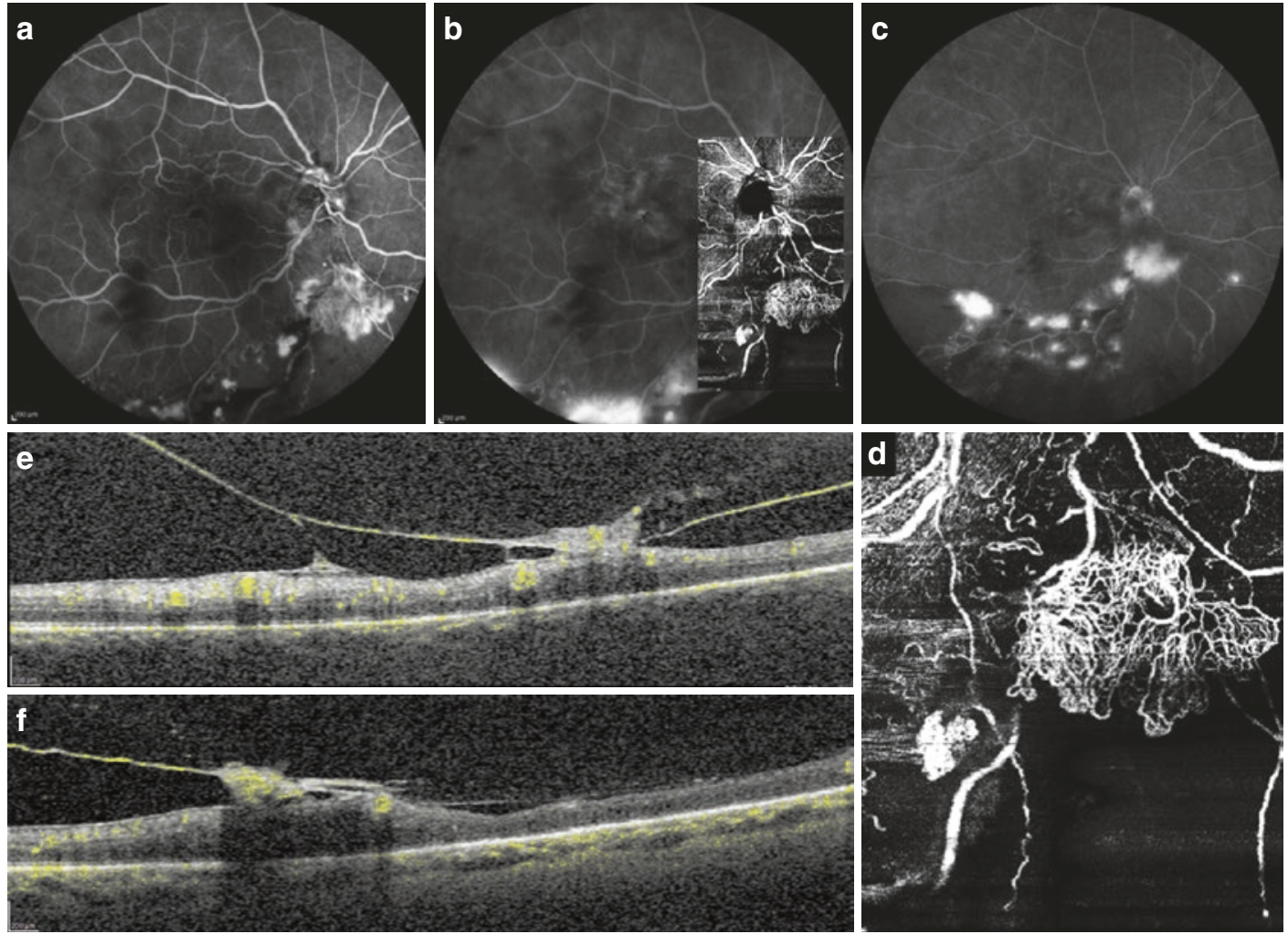

changes, areas of non-perfusion and neovascularizations.

Fig. 6.12 Multimodal imaging of retinal vein occlusion. Fluorescein angiography $(\mathbf{a}-\mathbf{c})$ and optical coherence tomography angiography (b, d) showing vascular

sized that the primary involvement is the alteration of Müller cells and secondary the vascular and tissue remodeling. Fluorescein angiography displays the capillary telangiectasia with dilated and blunted retinal venules at right angles into the temporal parafoveolar area.

OCTA shows the prominent right-angle veins with the distortion of the foveal avascular zone with cavitations. Idiopathic macular telangiectasia could be complicated by retinal choroidal anastomosis, visible in indocyanine green angiography and OCTA (Fig. 6.13).

\subsubsection{Age Related Macular Degeneration}

Several studies have shown the utility of OCTA in the diagnosis and monitoring of age related macular degeneration (AMD). The advent of
The B-Scans (e, f) reveal the retinal neovascularizations projecting into the vitreous space

OCTA offered the opportunity to non-invasively visualize the neovascular networks and correlate the OCTA appearance with the standard imaging techniques and observe new findings.

In this context, age related macular degeneration is characterized by different neovascular lesions. Type 1 neovascularization occurs between the retinal pigment epithelium and Bruch's membrane, while Type 2 neovascularization is characterized by the growth of the neovascular tissue through the retinal pigment epithelium-Bruch's membrane-choriocapillaris complex to the subretinal space [50, 51]. OCTA is able to show the neovascular network with a similar appearance to indocyanine green angiography. However, indocyanine green angiography is a dynamic examination that may reveal the feeder vessel of the lesion, while OCTA is not able to provide a dynamic representation of blood flow and to detect the feeder vessel (Fig. 6.14, 6.15, 6.16, and 6.17). 

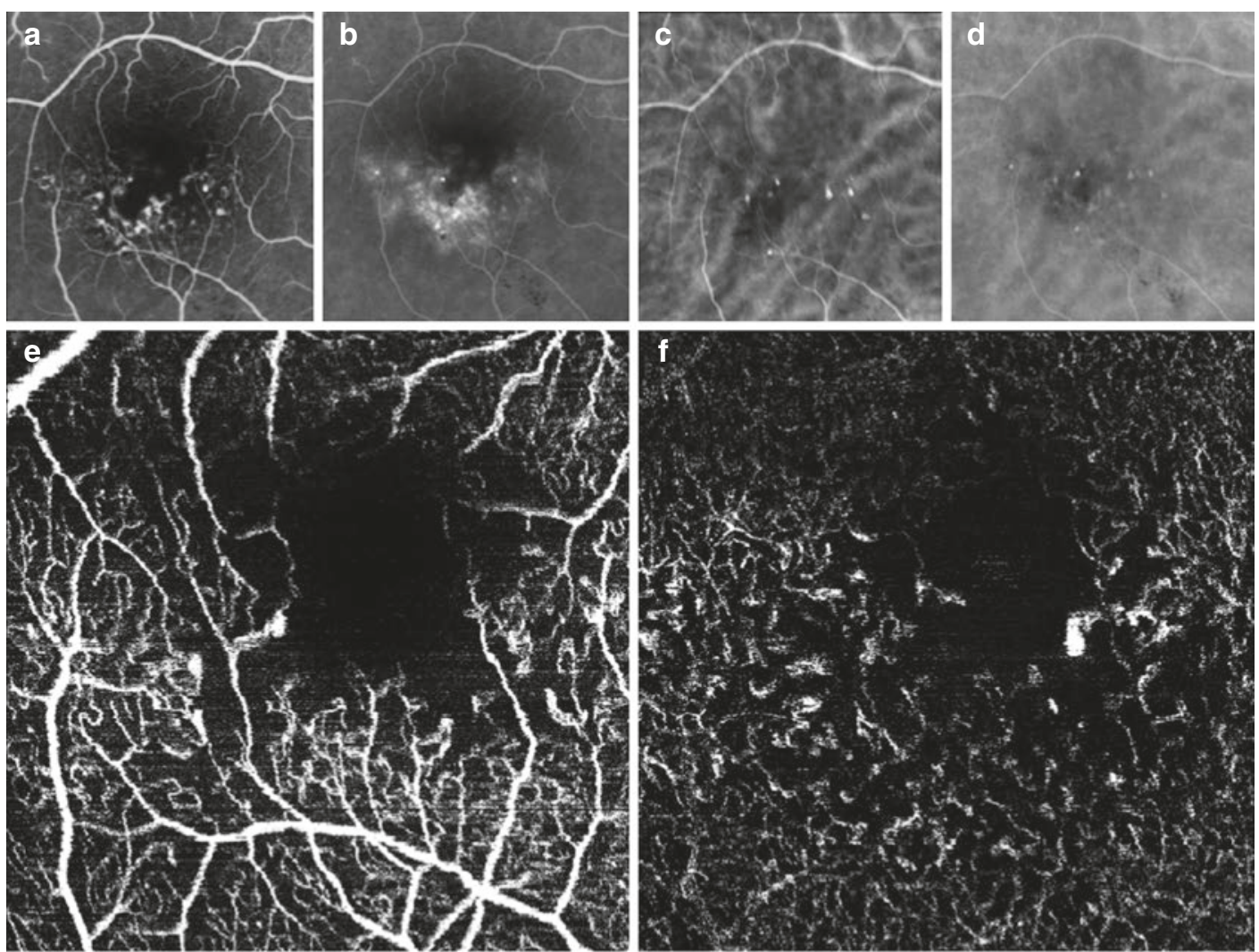

g

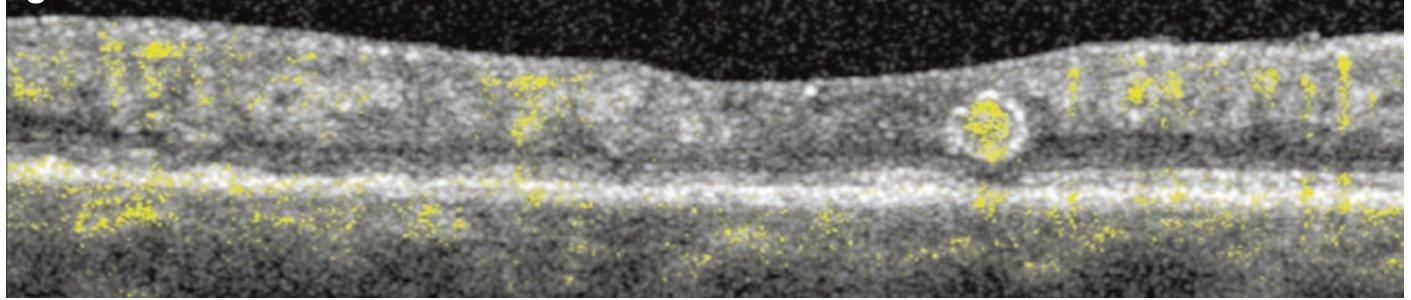

Fig. 6.13 Multimodal imaging of Type I idiopathic macular telangiectasia. Fluorescein angiography $(\mathbf{a}, \mathbf{b})$ and indocyanine green angiography $(\mathbf{c}, \mathbf{d})$ showing the dilated telangiectatic perifoveal vessels with leakage in the late

In the context of Type 1 lesions, focal polypoidal changes of the neovascular tissue could be found [52]. The gold standard to detect polypoidal lesions is indocyanine green angiography where single or multiple focal nodular areas of hyperfluorescence are arising from the choroidal circulation with or without an associated branching vascular network. The ability to detect polypoidal lesions by OCTA is not well defined. In the majority of cases, OCTA is not able to show the phase. OCTA at the superficial retinal capillary plexus (e) and deep (f) retinal capillary plexus revealing the dilated telangiectatic perifoveal vessels. The B-Scan (g) displaying the dilated telangiectatic vessel with flow sign inside

lesion due to the low velocity of the blood flow inside and then the final appearance is an area of absence of signal (Fig. 6.18).

Type 3 neovascularization may originate from both circulations simultaneously as initial focal retinal proliferation and progression, or focal retinal proliferation with preexisting or simultaneous choroidal proliferation, or initial focal choroidal proliferation and progression [53]. Type 3 neovascularization can be visualized as a discrete 

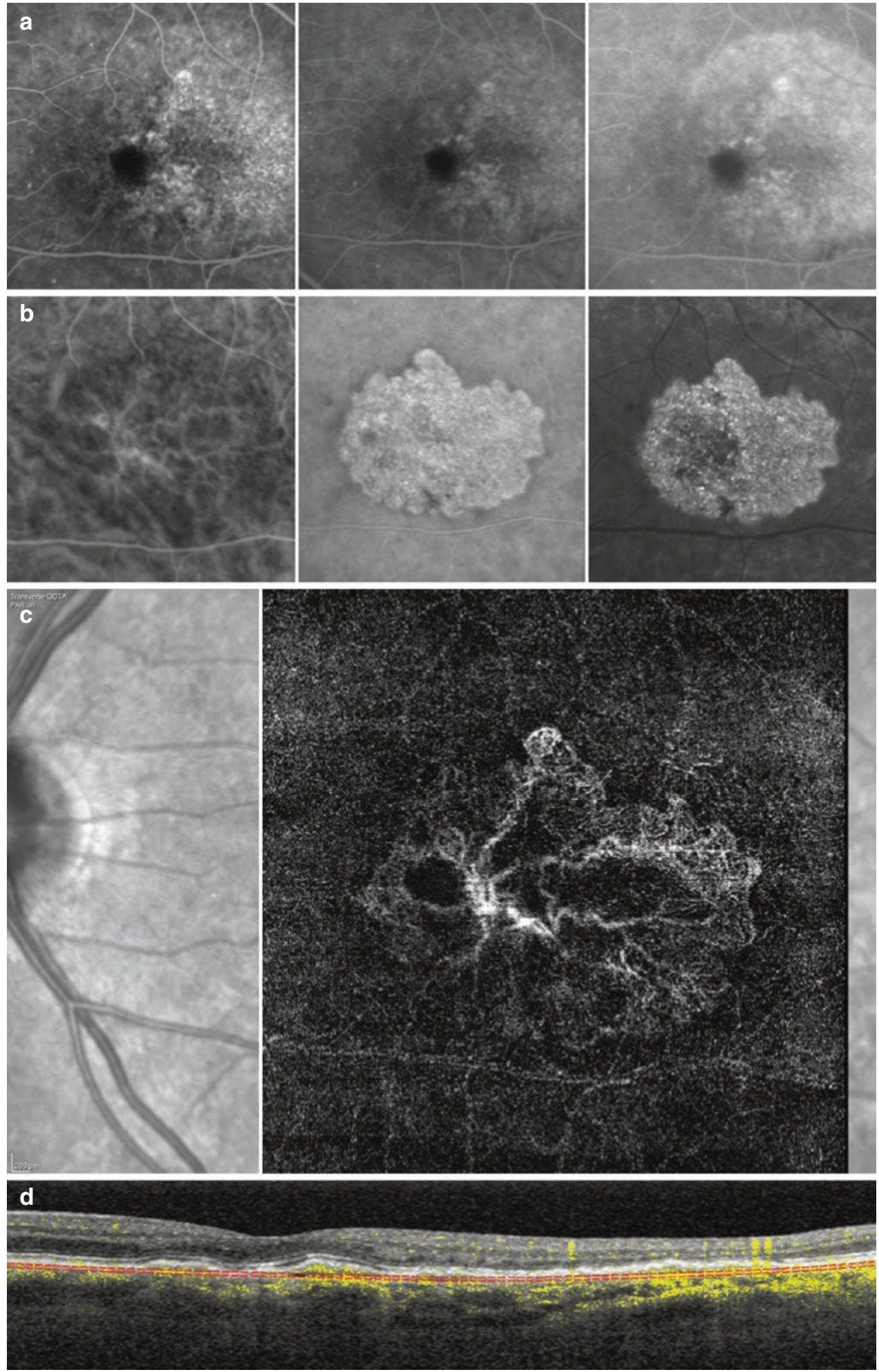

Fig. 6.14 Multimodal imaging of Type 1 neovascularization. Early to late phase of fluorescein angiography (a) showing pinpoints of hyperfluorescence. Early to late phase of indocyanine green angiography (b) revealing central hyperfluorescent area corresponding to type 1 neovascularization. Optical coherence tomography angiography (c) displaying a well-defined neovascular network under the retinal pigment epithelium (d) 

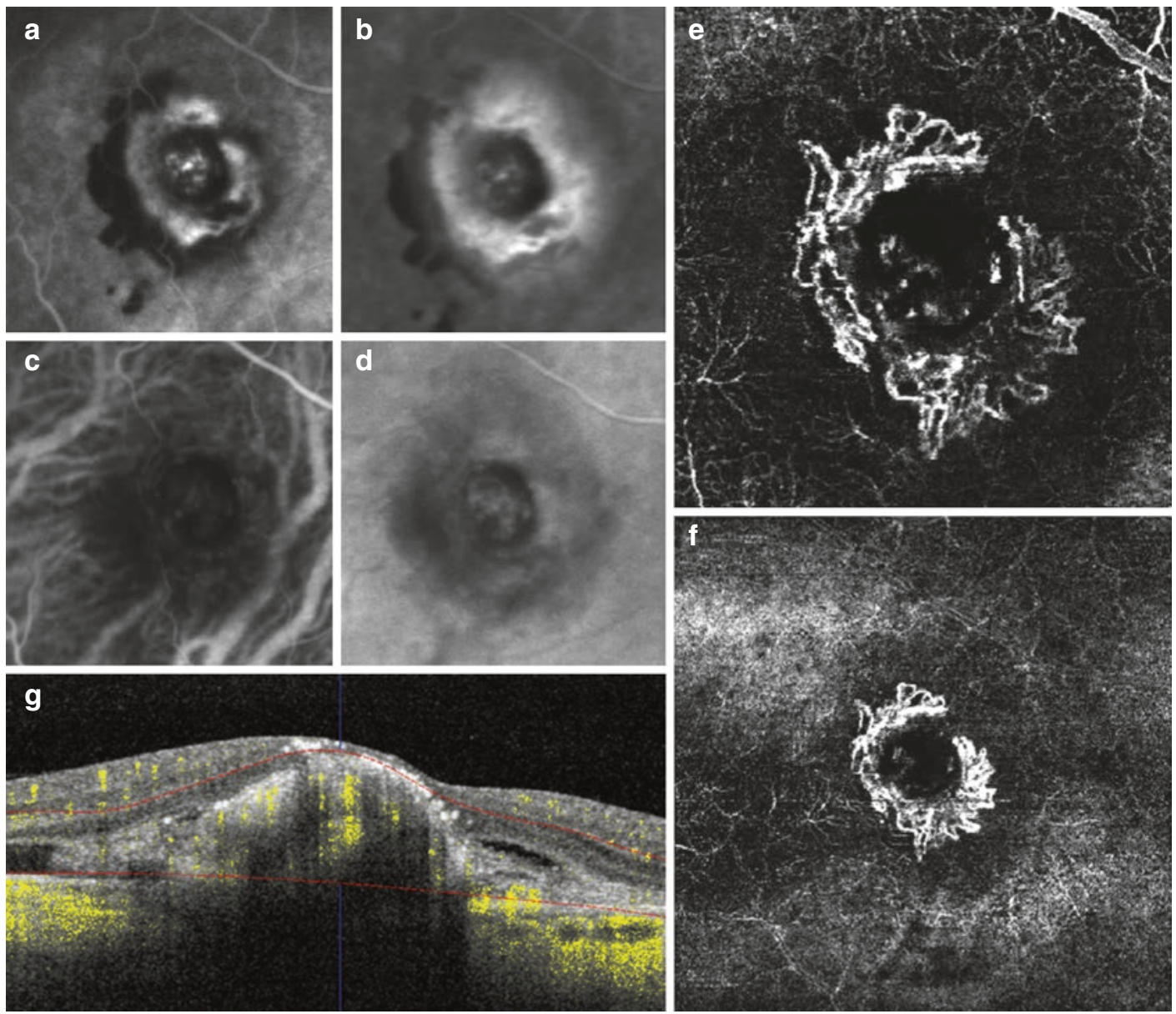

Fig. 6.15 Multimodal imaging of Type 2 neovascularization. Early (A) phase of fluorescein angiography showing a well-defined neovascular network with leakage in the late phase (b). Early (c) and late (d) phases of indocyanine green angiography revealing the neovascular network. Optical coherence tomography angiography in the

high flow linear structure extending from the middle retinal layers into the deep retina, which sometimes extent throughout the retinal pigment epithelium on OCTA. In Fig. 6.19 OCTA reveals a branching vessel anastomoses with the deep retinal capillary plexus and goes into the outer retina and eventually into the subretinal pigment epithelium space.

In contrast, geographic atrophy (GA) is a well-established end-stage manifestation of AMD [54, 15]. It results from the degeneration of photoreceptors, retinal pigment epithelium, and choriocapillaris. In this context OCTA is a very useful imaging modality to observe the
$3 \times 3 \mathrm{~mm}(\mathbf{e})$ and $6 \times 6 \mathrm{~mm}(\mathbf{f})$ showing the neovascular network with clearly visible and defined margins. Optical coherence tomography (g) displaying the detachment of retinal pigment epithelium with subretinal hyperreflective material and subretinal fluid

presence of $\mathrm{CNV}$ at the peripheral border of atrophy. In fact, the ability to detect neovascularization with the standard angiographic examination could be challenging due to the alteration of retinal pigment epithelium and exposure of normal choroidal vessels. In this context, OCTA is a very useful technique that is able to show the neovascular network with the appropriate segmentation (Fig. 6.20). Moreover, OCTA was used to evaluate the status of choriocapillaris in patients with GA. A general loss of choriocapillaris flow associated with drusen and subretinal drusenoid deposits was found in OCTA. 

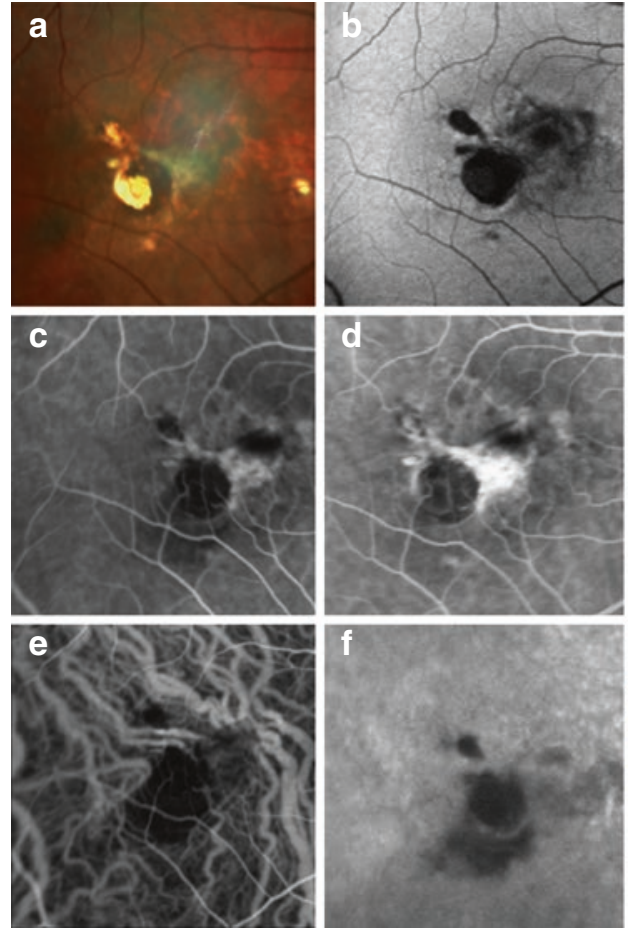

Fig. 6.16 Multimodal imaging of Type 2 neovascularization secondary to pathologic myopia. MultiColor imaging (a) and blue autofluorescence (b) showing fundus abnormalities related to pathologic myopia. Early phase (c) and late phase of fluorescein angiography (d) revealing the Type 2 neovascularization as an hyperfluorescent area that become
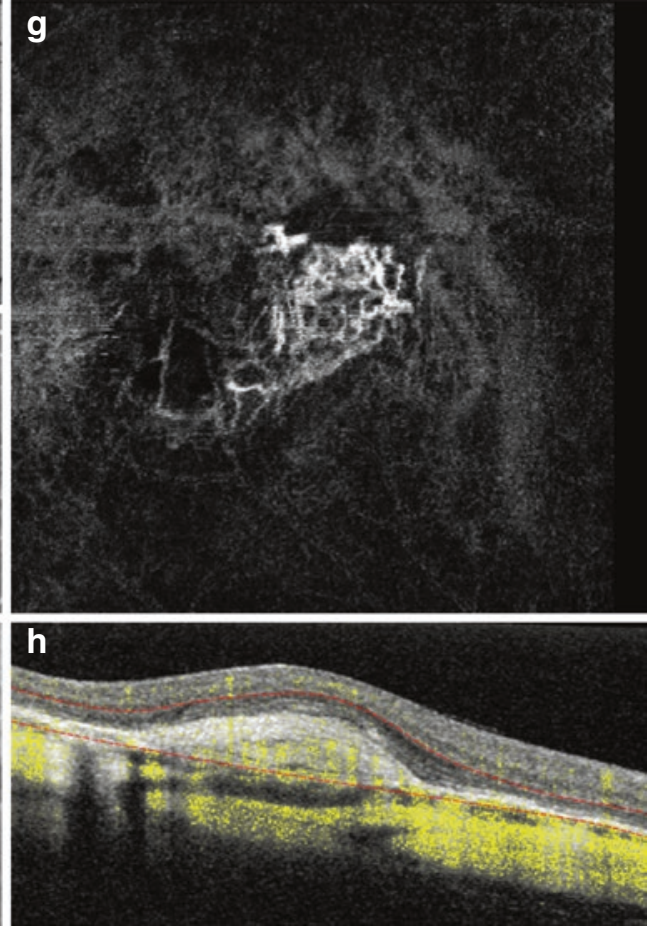

more intense with moderate leakage in the late phase (d). Early phase (e), late phase (f) of indocyanine green angiography and OCTA (g) displaying the neovascular network with well circumscribed appearance at the border of atrophy. OCT/OCTA B-Scan (h) showing the subretinal hyperreflective material corresponding to the neovascular lesion
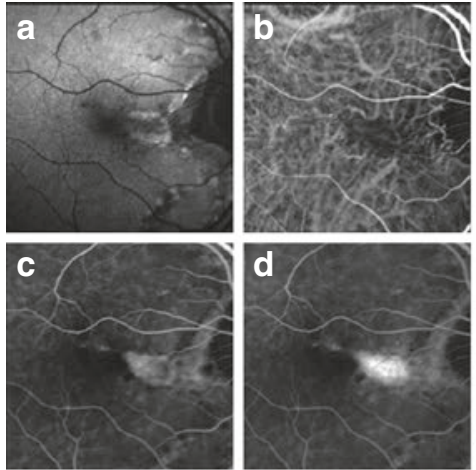

e

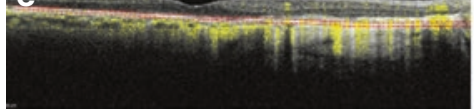

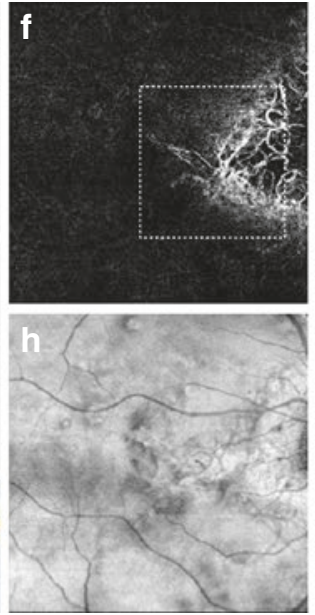

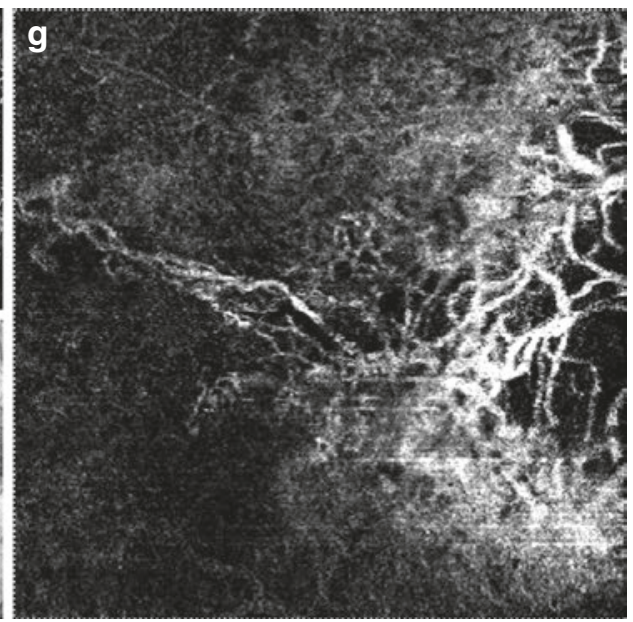

Fig. 6.17 Multimodal imaging of Type 2 neovascularization secondary to angioid streaks. Blue autofluorescence (a) revealing the fundus alterations secondary to angioid streaks. Indocyanine green angiography (b) and fluorescein angiography (c) showing an hyperfluorescent area that become more intense with moderate leakage in the late phase (d), as Type 2 neovascularization. Optical coherence tomography displaying the area of atrophy with the neovascular tissue above the retinal pigment epithelium without sub/intraretinal fluid (e). Optical coherence tomography angiography in the $6 \times 6 \mathrm{~mm}(\mathbf{f})$ and $3 \times 3 \mathrm{~mm}(\mathrm{~g})$ showing a choroidal neovascularization with a defined network that closely follows the trajectory of the angioid streak-well appreciable on the en face optical coherence tomography (h) 

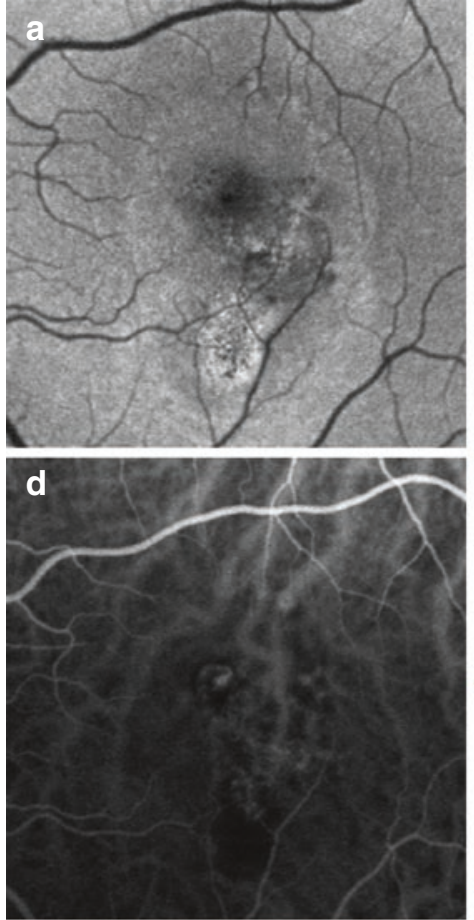

f

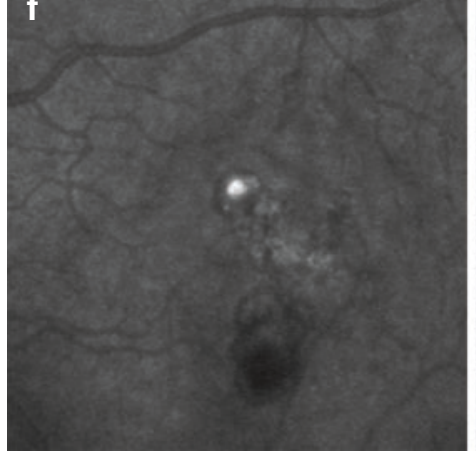

Fig. 6.18 Multimodal imaging of polypoidal neovascularization. Fundus autofluorescence (a) showing diffuse alteration of retinal pigment epithelium and areas of atrophy. Fluorescein angiography $(\mathbf{b}, \mathbf{c})$ revealing a diffuse hyperfluorescence and hypofluorescence points. Indocyanine green angiography in the different phases
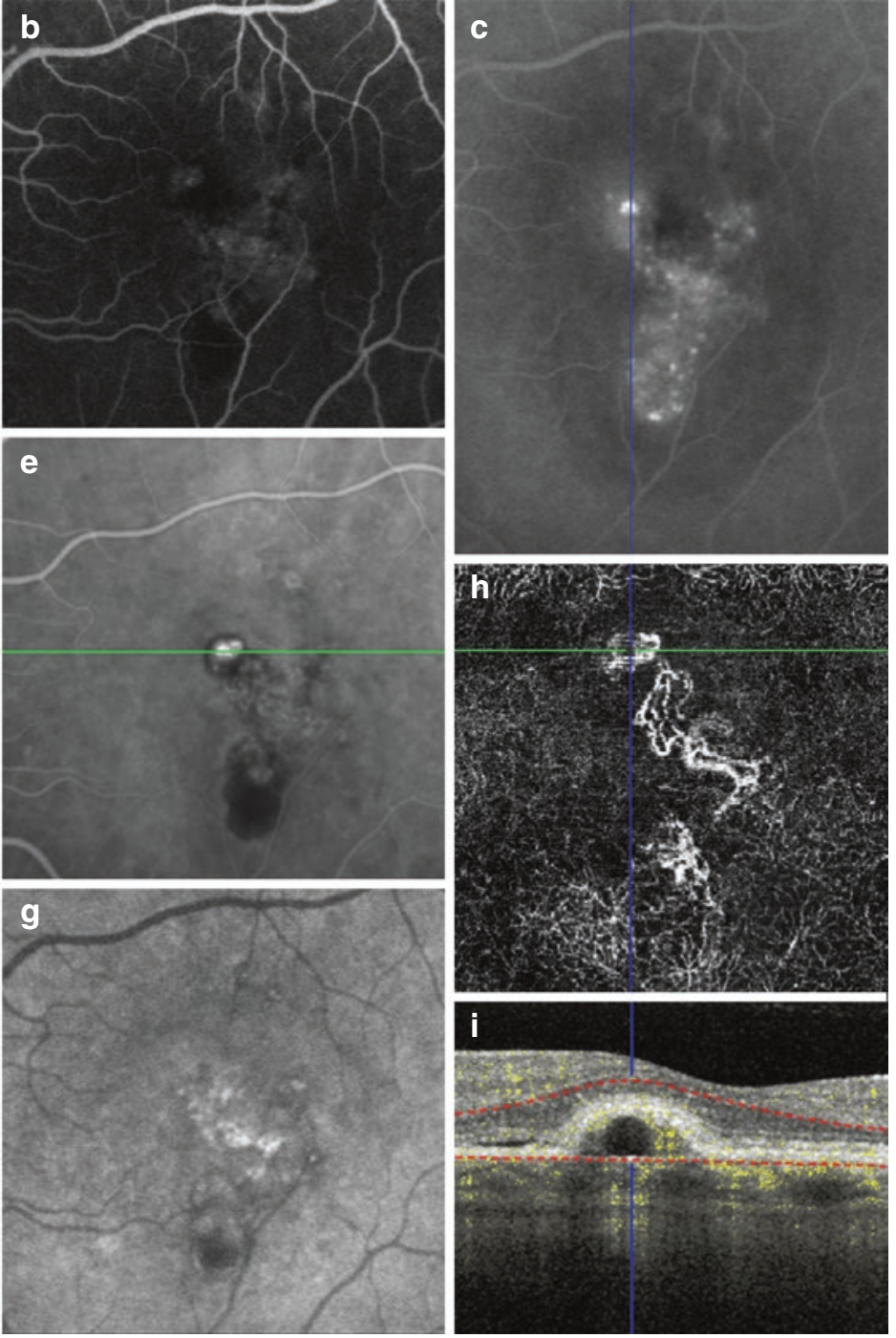

$(\mathbf{d}-\mathbf{g})$ revealing the Type 1 neovascular network with hyperfluorescent polypoidal lesion. OCTA $(\mathbf{h}, \mathbf{i})$ showing the central neovascular network, as Type 1 neovascularization, one polypoidal lesion but not the polypoidal lesion in the temporal area 

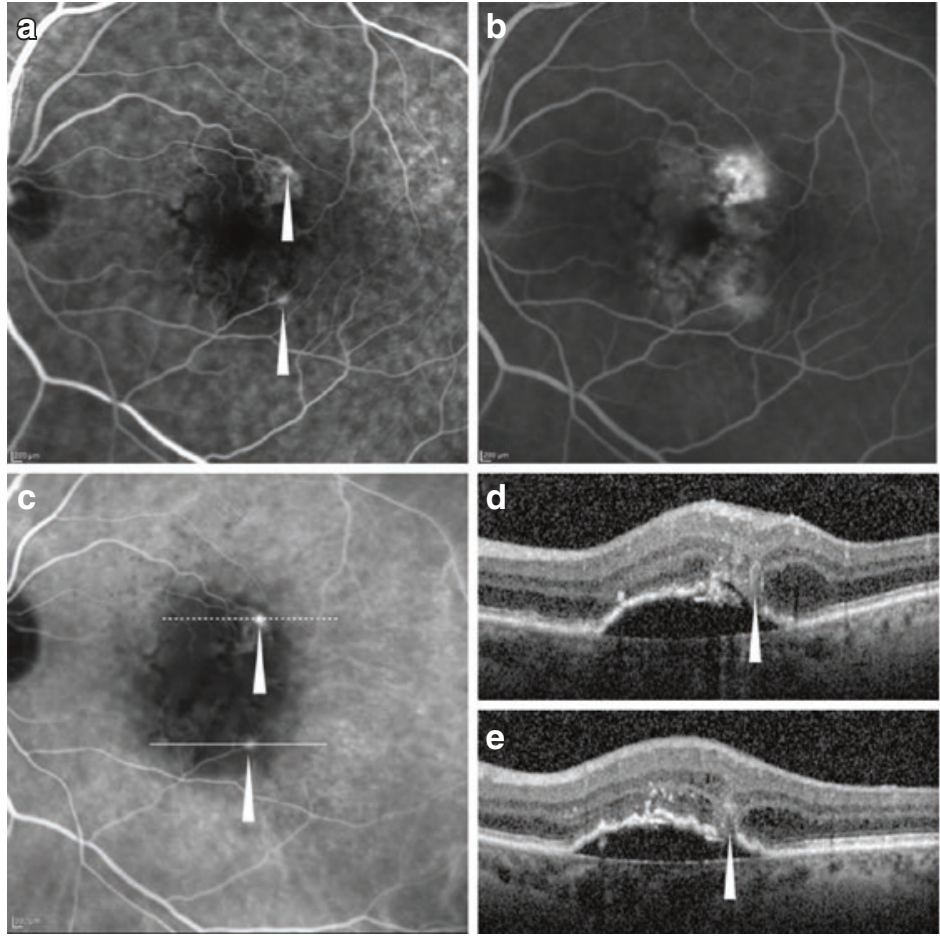
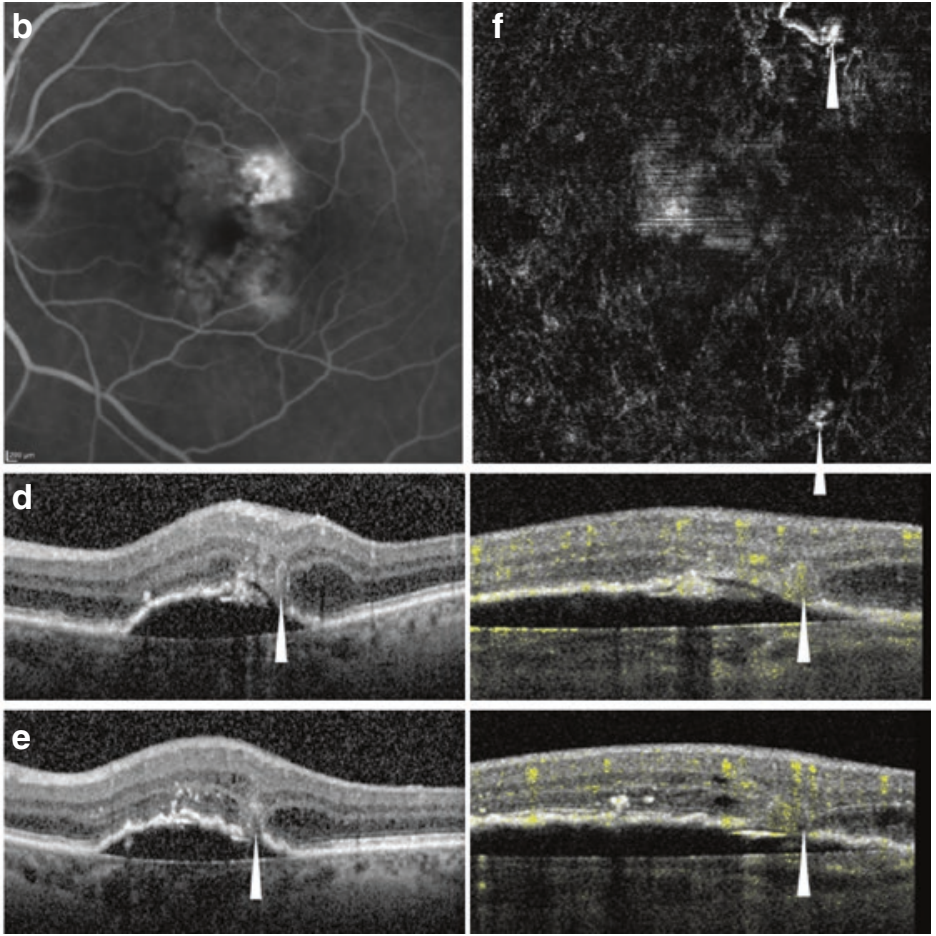

Fig.6.19 Multimodal imaging of Type 3 neovascularization. Fluorescein angiography $(\mathbf{a}, \mathbf{b})$ and indocyanine green angiography (c) revealing the neovascularizations (arrowheads) as two round hyperfluorescent points with leakage in the late phases. The two white lines indicate the exact location of the optical coherence tomography sec- tions (d, e) showing the detachment of retinal pigment epithelium with intraretinal cystoid space. Optical coherence tomography angiography (f) revealing a tuft-shaped, high-flow lesion (open arrowheads) in the outer retinal layers abutting into the sub-retinal pigment epithelium space
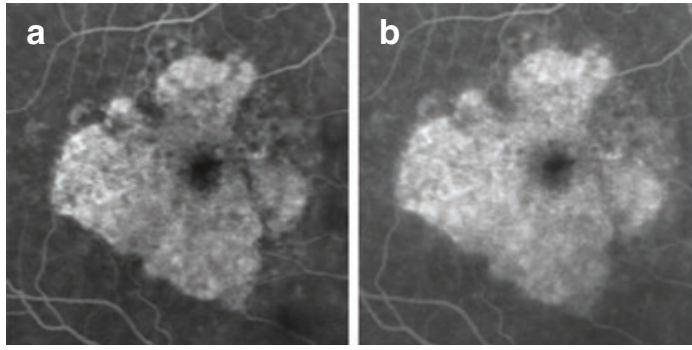

Fig. 6.20 Multimodal imaging of geographic atrophy. Fluorescein angiography $(\mathbf{a}, \mathbf{b})$ revealing the central area of atrophy as a hyperfluorescence area with staining. Indocyanine green angiography $(\mathbf{c}, \mathbf{d})$ showing well evidently the medium-large choroidal vessels under the atrophic area. En face optical coherence tomography (e) and optical coherence tomography angiography (f) showing
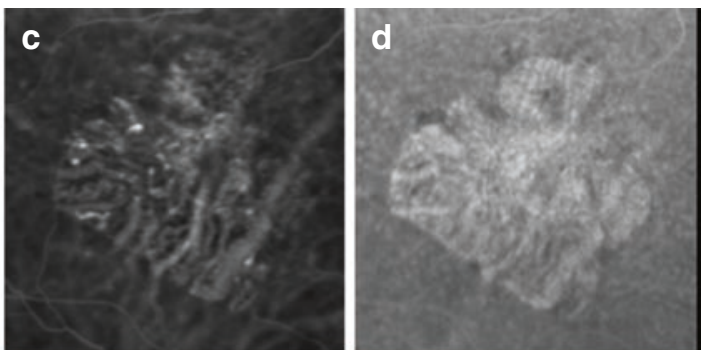

the area of atrophy with rarefied choriocapillaris and Sattler layer. Optical coherence tomography $(\mathbf{g}, \mathbf{h})$ displaying hypertransmission of the signal below the level of the retinal pigment epithelium and into the choroid resulting from loss of scatter or attenuation from overlying retinal pigment epithelium and neurosensory retina 

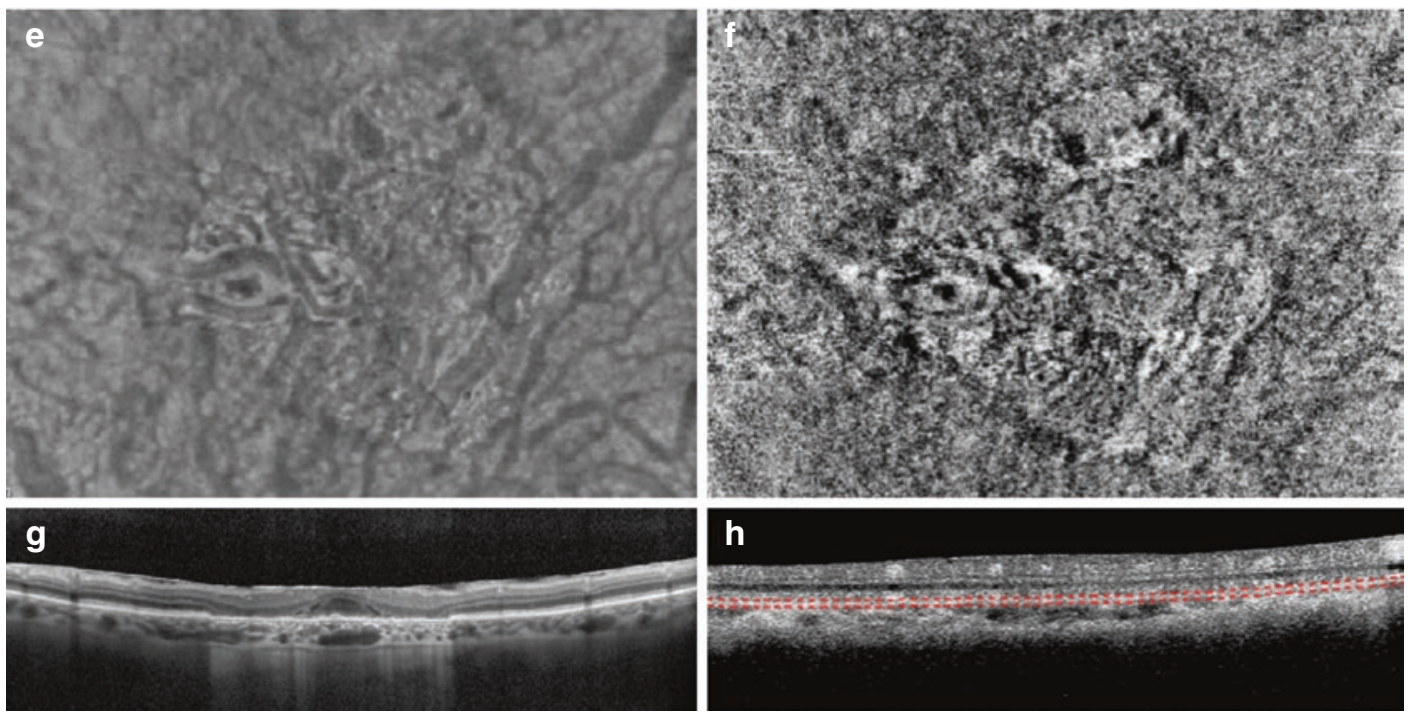

h

Fig. 6.20 (continued)

\subsection{Conclusion}

In this article the basic principles, major sources of artifacts and the clinical application of OCTA were discussed. The discussion of the clinical examples showed that OCTA provides diagnostic value in several vascular diseases of the eye. However, the current state of OCTA has not yet fully replaced the gold standard dye-based angiography because of important limitations.

Ongoing endeavors to improve OCTA are addressing these shortcomings.

This includes targeting greater fields of view, which is especially important in DR. Improvements to automatic segmentation algorithms in the context of pathological alterations are necessary for reliable results. Based on these future improvements it is expected that robust metrics and sensitive monitoring of disease progression can be achieved.

\section{References}

1. Spaide RF, Klancnik JM Jr, Cooney MJ. Retinal vascular layers imaged by fluorescein angiography and optical coherence tomography angiography. JAMA Ophthalmol. 2015;133(1):45-50.

2. Matsunaga D, Yi J, Puliafito CA, Kashani AH. OCT angiography in healthy human subjects. Ophthalmic Surg Las Imag Retin. 2014;45(6):510-5.

3. Tan PE, Yu PK, Balaratnasingam C, Cringle SJ, Morgan WH, McAllister IL, Yu DY. Quantitative confocal imaging of the retinal microvasculature in the human retina. Invest Ophthalmol Vis Sci. 2012;53(9):5728-36.

4. Zhang A, Zhang Q, Chen CL, Wang RK. Methods and algorithms for optical coherence tomography-based angiography: a review and comparison. J Biomed Opt. 2015;20(10):100901.

5. Spaide RF, Fujimoto JG, Waheed NK. Image artifacts in optical coherence tomography angiography. Retina. 2015;35(11):2163-80.

6. Johnson RN, Fu AD, McDonald HR, Jumper JM, Ai E, Cunningham ET, Lujan BJ. Fluorescein angiography: basic principles and interpretation. In: Retina. 5th ed. Amsterdam: Elsevier Inc; 2012.

7. Spaide RF, Fujimoto JG, Waheed NK, Sadda SR, Staurenghi G. Optical coherence tomography angiography. Prog Retin Eye Res. 2018;64:1-55.

8. Riva CE, Grunwald JE, Sinclair SH, Petrig B. Blood velocity and volumetric flow rate in human retinal vessels. Invest Ophthalmol Vis Sci. 1985;26(8):1124-32.

9. Vakoc BJ, Tearney GJ, Bouma BE. Statistical properties of phase-decorrelation in phase-resolved Doppler optical coherence tomography. IEEE Trans Med Imaging. 2009;28(6):814-21.

10. Cheng Y, Guo L, Pan C, Lu T, Hong T, Ding Z, Li P. Statistical analysis of motion contrast in optical coherence tomography angiography. J Biomed Opt. 2015;20(11):116004.

11. Braaf B, Donner S, Nam AS, Bouma BE, Vakoc BJ. Complex differential variance angiography with 
noise-bias correction for optical coherence tomography of the retina. Biomed Opt Express. 2018;9(2):486.

12. Chan G, Balaratnasingam C, Yu PK, Morgan WH, McAllister IL, Cringle SJ, Yu DY. Quantitative morphometry of perifoveal capillary networks in the human retina. Invest Ophthalmol Vis Sci. 2012;53(9):5502-14.

13. Campbell JP, Zhang M, Hwang TS, Bailey ST, Wilson DJ, Jia Y, Huang D. Detailed vascular anatomy of the human retina by projection-resolved optical coherence tomography angiography. Sci Rep. 2017;7:42201.

14. Hirano T, Chanwimol K, Weichsel J, Tepelus T, Sadda S. Distinct retinal capillary plexuses in normal eyes as observed in optical coherence tomography angiography axial profile analysis. Sci Rep. 2018;8(1):9380.

15. Holz FG, Strauss EC, Schmitz-Valckenberg S, van Lookeren Campagne M. Geographic atrophy: clinical features and potential therapeutic approaches. Ophthalmology. 2014;121(5):1079-91.

16. Rocholz R, Teussink MM, Dolz-Marco R, Holzhey C, Dechent J, Tafreshi A, Schulz S. SPECTRALIS optical coherence tomography angiography (OCTA): principles and clinical applications. Heidelberg Engineering Academy. 2018. https://academy.heidelbergengineering.com/course/view.php?id=505.

17. Agemy SA, Scripsema NK, Shah CM, Chui T, Garcia PM, Lee JG, Gentile RC, Hsiao YS, Zhou Q, Ko T, Rosen RB. Retinal vascular perfusion density mapping using optical coherence tomography angiography in normals and diabetic retinopathy patients. Retina. 2015;35(11):2353-63.

18. Chu Z, Lin J, Gao C, Xin C, Zhang Q, Chen CL, Roisman L, Gregori G, Rosenfeld PJ, Wang RK. Quantitative assessment of the retinal microvasculature using optical coherence tomography angiography. J Biomed Opt. 2016;21(6):66008.

19. Jia Y, Bailey ST, Hwang TS, McClintic SM, Gao SS, Pennesi ME, Flaxel CJ, Lauer AK, Wilson DJ, Hornegger J, Fujimoto JG, Huang D. Quantitative optical coherence tomography angiography of vascular abnormalities in the living human eye. Proc Natl Acad Sci U S A. 2015;112(18):E2395-402.

20. Jia Y, Morrison JC, Tokayer J, Tan O, Lombardi L, Baumann B, Lu CD, Choi W, Fujimoto JG, Huang D. Quantitative OCT angiography of optic nerve head blood flow. Biomed Opt Express. 2012;3(12):3127-37.

21. Uji A, Balasubramanian S, Lei J, Baghdasaryan E, Al-Sheikh M, Sadda SR. Choriocapillaris imaging using multiple en face optical coherence tomography angiography image averaging. JAMA Ophthalmol. 2017;135(11):1197-204.

22. Lu Y, Simonett JM, Wang J, Zhang M, Hwang T, Hagag AM, Huang D, Li D, Jia Y. Evaluation of automatically quantified foveal avascular zone metrics for diagnosis of diabetic retinopathy using optical coherence tomography angiography. Invest Ophthalmol Vis Sci. 2018;59(6):2212-21.

23. Coscas F, Cabral D, Pereira T, Geraldes C, Narotamo H, Miere A, Lupidi M, Sellam A, Papoila A, Coscas G, Souied E. Quantitative optical coherence tomography angiography biomarkers for neovascular age- related macular degeneration in remission. PLoS One. 2018;13(10):e0205513.

24. Zhang Q, Chen CL, Chu Z, Zheng F, Miller A, Roisman L, Rafael de Oliveira Dias J, Yehoshua Z, Schaal KB, Feuer W, Gregori G, Kubach S, An L, Stetson PF, Durbin MK, Rosenfeld PJ, Wang RK. Automated quantitation of choroidal neovascularization: a comparison study between spectraldomain and swept-source OCT angiograms. Invest Ophthalmol Vis Sci. 2017;58(3):1506-13.

25. Johannesen SK, Viken JN, Vergmann AS, Grauslund J. Optical coherence tomography angiography and microvascular changes in diabetic retinopathy: a systematic review. Acta Ophthalmol. 2019;97:7.

26. Tsai G, Banaee T, Conti FF, Singh RP. Optical coherence tomography angiography in eyes with retinal vein occlusion. J Ophthalmic Vis Res. 2018;13(3):315-32.

27. Dimitrova G, Chihara E. Implication of deep-vascularlayer alteration detected by optical coherence tomography angiography for the pathogenesis of diabetic retinopathy. Ophthalmologica. 2019;241:179-82.

28. Corvi F, Pellegrini M, Erba S, Cozzi M, Staurenghi G, Giani A. Reproducibility of vessel density, fractal dimension, and foveal avascular zone using 7 different optical coherence tomography angiography devices. Am J Ophthalmol. 2018;186:25-31.

29. Lei J, Pei C, Wen C, Abdelfattah NS. Repeatability and reproducibility of quantification of superficial peri-papillary capillaries by four different optical coherence tomography angiography devices. Sci Rep. 2018;8(1):17866.

30. Rabiolo A, Gelormini F, Sacconi R, Cicinelli MV, Triolo G, Bettin P, Nouri-Mahdavi K, Bandello F, Querques G. Comparison of methods to quantify macular and peripapillary vessel density in optical coherence tomography angiography. PLoS One. 2018;13(10):e0205773.

31. Liu L, Gao SS, Bailey ST, Huang D, Li D, Jia Y. Automated choroidal neovascularization detection algorithm for optical coherence tomography angiography. Biomed Opt Express. 2015;6(9):3564-76.

32. Zhang M, Hwang TS, Campbell JP, Bailey ST, Wilson DJ, Huang D, Jia Y. Projection-resolved optical coherence tomographic angiography. Biomed Opt Express. 2016;7(3):816-28.

33. Lee K, Zhang $\mathrm{H}$, Wahle $\mathrm{A}$, Abràmoff $\mathrm{MD}$, Sonka M. Multi-layer 3D simultaneous retinal OCT layer segmentation: just-enough interaction for routine clinical use. Cham: Springer International Publishing; 2018. p. 862-71.

34. Jia Y, Tan O, Tokayer J, Potsaid B, Wang Y, Liu JJ, Kraus MF, Subhash H, Fujimoto JG, Hornegger J, Huang D. Split-spectrum amplitude-decorrelation angiography with optical coherence tomography. Opt Express. 2012;20(4):4710-25.

35. Kraus M, Mayer MA, Bock R, Potsaid B, Manjunath V, Duker JS, Hornegger J, Fujimoto JG. Motion artifact correction in OCT volume scans using image registration. Invest Ophthalmol Vis Sci. 2010;51(13):4405.

36. Kraus MF, Potsaid B, Mayer MA, Bock R, Baumann B, Liu JJ, Hornegger J, Fujimoto JG. Motion correc- 
tion in optical coherence tomography volumes on a per A-scan basis using orthogonal scan patterns. Biomed Opt Express. 2012;3(6):1182-99.

37. Fercher AF, Drexler W, Hitzenberger CK, Lasser T. Optical coherence tomography-principles and applications. Rep Prog Phys. 2003;66(2):239.

38. Gass JD. A fluorescein angiographic study of macular dysfunction secondary to retinal vascular disease. V. Retinal telangiectasis. Arch Ophthalmol. 1968;80(5):592-605.

39. Marmor MF, Ravin JG. Fluorescein angiography: insight and serendipity a half century ago. Arch Ophthalmol. 2011;129(7):943-8.

40. Ishibazawa A, Nagaoka T, Takahashi A, Omae T, Tani T, Sogawa K, Yokota H, Yoshida A. Optical coherence tomography angiography in diabetic retinopathy: a prospective pilot study. Am J Ophthalmol. 2015;160(1):35-44 e1.

41. Salz DA, de Carlo TE, Adhi M, Moult E, Choi W, Baumal CR, Witkin AJ, Duker JS, Fujimoto JG, Waheed NK. Select features of diabetic retinopathy on swept-source optical coherence tomographic angiography compared with fluorescein angiography and normal eyes. JAMA Ophthalmol. 2016;134(6):644-50.

42. Klein R, Klein BE, Moss SE, Meuer SM. The epidemiology of retinal vein occlusion: the Beaver Dam Eye Study. Trans Am Ophthalmol Soc. 2000;98:13341; discussion 141-3.

43. Rehak J, Rehak M. Branch retinal vein occlusion: pathogenesis, visual prognosis, and treatment modalities. Curr Eye Res. 2008;33(2):111-31.

44. Hayreh SS, Zimmerman MB, Podhajsky P. Incidence of various types of retinal vein occlusion and their recurrence and demographic characteristics. Am J Ophthalmol. 1994;117(4):429-41.

45. Wilkinson CP, Ferris FL III, Klein RE, Lee PP, Agardh CD, Davis M, Dills D, Kampik A, Pararajasegaram R, Verdaguer JT. Global Diabetic Retinopathy Project,
G., Proposed international clinical diabetic retinopathy and diabetic macular edema disease severity scales. Ophthalmology. 2003;110(9):1677-82.

46. Gass JD, Oyakawa RT. Idiopathic juxtafoveolar retinal telangiectasis. Arch Ophthalmol. 1982;100(5):769-80.

47. Gass JD, Blodi BA. Idiopathic juxtafoveolar retinal telangiectasis. Update of classification and follow-up study. Ophthalmology. 1993;100(10):1536-46.

48. Yannuzzi LA, Bardal AM, Freund KB, Chen KJ, Eandi CM, Blodi B. Idiopathic macular telangiectasia. Arch Ophthalmol. 2006;124(4):450-60.

49. Wong WT, Forooghian F, Majumdar Z, Bonner RF, Cunningham D, Chew EY. Fundus autofluorescence in type 2 idiopathic macular telangiectasia: correlation with optical coherence tomography and microperimetry. Am J Ophthalmol. 2009;148(4):573-83.

50. Gass J. Biomicroscopic and histopathologic considerations regarding the feasibility of surgical excision of subfoveal neovascular membranes. Trans Am Ophthalmol Soc. 1994;92:91.

51. Gass JDM. Stereoscopic atlas of macular diseases: diagnosis and treatment (2 Volume Set). St. Louis, MO: Mosby; 1997.

52. Yannuzzi LA, Sorenson J, Spaide RF, Lipson B. Idiopathic polypoidal choroidal vasculopathy (IPCV) 1990. Retina. 2012;32(Suppl 1):1-8.

53. Freund KB, Zweifel SA, Engelbert M. Do we need a new classification for choroidal neovascularization in age-related macular degeneration? Retina. 2010;30:1333.

54. Ferris FL III, Wilkinson C, Bird A, Chakravarthy U, Chew E, Csaky K, Sadda SR, Committee BI. f. M. R. C., Clinical classification of age-related macular degeneration. Ophthalmology. 2013;120(4):844-51. 
Open Access This chapter is licensed under the terms of the Creative Commons Attribution 4.0 International License (http://creativecommons.org/licenses/by/4.0/), which permits use, sharing, adaptation, distribution and reproduction in any medium or format, as long as you give appropriate credit to the original author(s) and the source, provide a link to the Creative Commons license and indicate if changes were made.

The images or other third party material in this chapter are included in the chapter's Creative Commons license, unless indicated otherwise in a credit line to the material. If material is not included in the chapter's Creative Commons license and your intended use is not permitted by statutory regulation or exceeds the permitted use, you will need to obtain permission directly from the copyright holder.

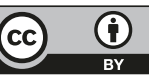

